\title{
ON THE STRUCTURE OF NEARLY PSEUDO-KÄHLER MANIFOLDS
}

\author{
LARS SCHÄFER
}

\begin{abstract}
Firstly we give a condition to split off the Kähler factor from a nearly pseudoKähler manifold and apply this to get a structure result in dimension 8. Secondly we extend the construction of nearly Kähler manifolds from twistor spaces to negatively curved quaternionic Kähler manifolds and para-quaternionic Kähler manifolds. The class of nearly pseudo-Kähler manifolds obtained from this construction is characterized by a holonomic condition. The combination of these results enables us to give a classification result in (real) dimension 10. Moreover, we show that a strict nearly pseudo-Kähler six-manifold is Einstein.
\end{abstract}

\section{INTRODUCTION}

Nearly Kähler geometry was introduced and studied in a series of papers by A. Gray in the seventies in the context of weak holonomy. To our best knowledge he only considers pseudoRiemannian metrics in his paper on 3-symmetric spaces [13. In the analysis of Killing spinors on pseudo-Riemannian manifolds [14] nearly pseudo-Kähler and nearly para-Kähler manifolds appear in a natural way. Levi-Civita flat nearly Kähler manifolds provide a special class of solutions of the topological-antitopological fusion equations on the tangent bundle [24, 25]. There is a strong similarity to special Kähler geometry. For these reasons we became interested in Levi-Civita flat nearly Kähler manifolds and were able to give a constructive classification [8, 9]. In particular it follows, that non-Kählerian examples only exist in pseudo-Riemannian geometry and that the real dimension is at least 12. In other words, nearly Kähler geometry in the pseudo-Riemannian world can be very different from the better-understood Riemannian world. In (real) dimension six nearly pseudo-Kähler manifolds satisfy an exterior system analogue to the Riemannian case. Details can be found in 23 . This system is used there to study such structures on products $G \times G$, where $G$ is a simple three-dimensional Lie group.

An interesting class of nearly Kähler manifolds $M^{4 n+2}$ can be constructed from twistor spaces over positive quaternionic Kähler manifolds. This class is characterized [5, 19] by the reducibility of the holonomy of the canonical connection $\bar{\nabla}$ to $U(n) \times U(1)$. We show in this article that the twistor spaces over negative quaternionic Kähler manifolds and para-quaternionic Kähler manifolds carry a nearly pseudo-Kähler structure and characterize the class of such examples by a holonomic condition.

Using this result, we classify nice and decomposable (cf. Definitions 2.7 and 2.10) nearly pseudoKähler manifolds in dimension ten.

Theorem A. Let $\left(M^{10}, J, g\right)$ be a nice decomposable nearly Kähler manifold, then the universal cover of $M$ is either the product of a pseudo-Kähler surface and a (strict) nearly pseudo-Kähler manifold $M^{6}$ or a twistor space of an eight-dimensional (para-)quaternionic Kähler manifold endowed with its canonical nearly pseudo-Kähler structure.

A strict nearly pseudo-Kähler six-manifold $M^{6}$ is shown to be Einstein in Theorem 2.11. In dimension eight simply connected strict nearly pseudo-Kähler manifolds are shown (Theorem 2.8) to be products $\Sigma \times M^{6}$ of a Riemannian surface $\Sigma$ and a strict nearly pseudo-Kähler manifold $M^{6}$.

Date: December 11, 2009.

2000 Mathematics Subject Classification. 53C15; 53C28; 53C29; 53C25; 53C26.

Key words and phrases. Nearly Kähler manifold, twistor spaces, pseudo-Riemannian metrics. 
In the first section of this paper we recall the definition of a nearly pseudo-Kähler manifold and generalize some facts and curvature identities to arbitrary signature. In the second section we give a general condition to split off the Kähler factor from a nearly pseudo-Kähler manifold, see Theorem 2.5. Using some linear algebra of three-forms this shows the splitting result for nice nearly pseudo-Kähler manifolds in dimension 8 . The argument also holds true for a Riemannian metric and gives an alternative proof for the known result. If a nice nearly pseudo-Kähler tenmanifold is in addition decomposable, we find two cases: In the first we can split off the Kähler factor and in the second the holonomy of $\bar{\nabla}$ is reducible with a complex one dimensional factor. This is one motivation to study twistor spaces. Before doing this in section four we recall some information on pseudo-Riemannian submersions in the third section. In the pseudo-Riemannian setting twistor spaces are a good source of examples, since quaternionic geometry is richer in negative scalar curvature than in positive (cf. Remark 4.7) and since we have the additional class of twistor spaces over para-quaternionic manifolds. In the last section we prove that a nearly pseudo-Kähler manifold $M$ of twistorial type (cf. Definition 5.11) is obtained from the above mentioned construction on a twistor space. This is done as follows: We prove that $M$ comes from a pseudo-Riemannian submersion $\pi: M \rightarrow N$. Then we use the nearly Kähler data on $M$ to endow $N$ with the structure of a (para-)quaternionic manifold. The proof is finished by identifying the twistor space of $N$ with $M$. The former proofs [5, 19] in the Riemannian case all use the inverse twistor construction of Penrose or LeBrun, which does not seem to be developed for the situations occurring in this text. As the reader might observe, the approach presented here holds also true for Riemannian metrics.

Acknowledgments. The author thanks Vicente Cortés for discussions.

\section{NEARLY PSEUdo-KÄHLER MANIFOLDS}

Definition 1.1. An almost pseudo-hermitian manifold $(M, J, g)$ is called nearly pseudo-Kähler manifold if it holds

$$
\left(\nabla_{X} J\right) X=0, \quad \forall X \in \Gamma(T M),
$$

where $\nabla$ is the Levi-Civita connection of the (pseudo-)Riemannian metric $g$. A nearly pseudoKähler manifold is called strict if it holds $\nabla_{X} J \neq 0$ for all $X \in T M$.

1.1. Curvature identities in the pseudo-Riemannian case. The starting point of a series of curvature identities are

$$
\begin{aligned}
R(W, X, Y, Z) & -R(W, X, J Y, J Z)=g\left(\left(\nabla_{W} J\right) X,\left(\nabla_{Y} J\right) Z\right), \\
R(W, X, W, Z) & +R(W, J X, W, J Z) \\
& -R(W, J W, X, J Z)=2 g\left(\left(\nabla_{W} J\right) X,\left(\nabla_{W} J\right) Z\right), \\
R(W, X, Y, Z) & =R(J W, J X, J Y, J Z),
\end{aligned}
$$

which were already proven for pseudo-Riemannian metrics by Gray [12. Let $\left\{e_{i}\right\}_{i=1}^{2 n}$ be a local orthonormal frame field, then the Ricci- and the Ricci*-tensor are given by

$$
g(\operatorname{Ric} X, Y)=\sum_{i=1}^{2 n} \epsilon_{i} R\left(X, e_{i}, Y, e_{i}\right), \quad g\left(\operatorname{Ric}^{*} X, Y\right)=\frac{1}{2} \sum_{i=1}^{2 n} \epsilon_{i} R\left(X, J Y, e_{i}, J e_{i}\right)
$$

with $\epsilon_{i}=g\left(e_{i}, e_{i}\right)=g\left(J e_{i}, J e_{i}\right)$ and $X, Y \in T M$. The frame $\left\{e_{i}\right\}_{i=1}^{2 n}$ is called adapted if it holds $J e_{i}=e_{i+n}$ for $i=1, \ldots, n$. Then it follows using an adapted frame from equations (1.2) and (1.3) that

$$
g(r X, Y):=g\left(\left(R i c-R i c^{*}\right) X, Y\right)=\sum_{i=1}^{2 n} \epsilon_{i} g\left(\left(\nabla_{X} J\right) e_{i},\left(\nabla_{Y} J\right) e_{i}\right) .
$$

Using the right hand-side we see

$$
[J, r]=0 .
$$

For the second derivative of the complex structure one has the identity

$$
2 g\left(\nabla_{W, X}^{2}(J) Y, Z\right)=-\sigma_{X, Y, Z} g\left(\left(\nabla_{W} J\right) X,\left(\nabla_{Y} J\right) J Z\right),
$$


which was proven in 12 for Riemannian metrics and holds true in the pseudo-Riemannian setting, cf. 14 Proposition 7.1. This identity implies

$$
\sum_{i=1}^{2 n} \epsilon_{i} \nabla_{e_{i}, e_{i}}^{2}(J) Y=-r(J Y)
$$

1.2. The canonical connection and some of its properties. An important property of nearly Kähler geometry is the existence of a canonical Hermitian connection [10] (see 8] for pseudoRiemannian metrics). This is the unique connection $\bar{\nabla}$ with skew-symmetric torsion, which parallelizes the metric $g$ and the almost complex structure $J$. Explicitely it is given by

$$
\bar{\nabla}_{X} Y=\nabla_{X} Y-\frac{1}{2} J\left(\nabla_{X} J\right) Y, \text { for } X, Y \in \Gamma(T M)
$$

and its torsion equals $\bar{T}(X, Y)=-J\left(\nabla_{X} J\right) Y$.

Proposition 1.2. Denote by $\bar{\nabla}$ the canonical connection of a nearly pseudo-Kähler manifold $(M, J, g)$. Then one has $\bar{\nabla}(T)=\bar{\nabla}(\nabla J)=0$.

Proof. The proof given in [5] essentially uses the explicit form (1.7) of the connection $\bar{\nabla}$ and the identity (1.5). Therefore the proposition generalizes to the pseudo-Riemannian case.

From Proposition 1.2 and the relation (1.7) of $\nabla$ and $\bar{\nabla}$ one obtains the following identities for the curvature tensor $\bar{R}$ of $\bar{\nabla}$ and the curvature tensor $R$ of the Levi-Civita connection $\nabla$

$$
\begin{aligned}
\bar{R}(W, X, Y, Z) & =R(W, X, Y, Z)-\frac{1}{2} g\left(\left(\nabla_{W} J\right) X,\left(\nabla_{Y} J\right) Z\right) \\
& +\frac{1}{4}\left[g\left(\left(\nabla_{W} J\right) Y,\left(\nabla_{X} J\right) Z\right)-g\left(\left(\nabla_{W} J\right) Z,\left(\nabla_{X} J\right) Y\right)\right] \\
& =\frac{1}{4}[3 R(W, X, Y, Z)+R(W, X, J Y, J Z) \\
& \left.+\sigma_{X Y Z} R(W, X, J Y, J Z)\right], \\
\bar{R}(W, J W, Y, J Z) & =\frac{1}{4}[5 R(W, J W, Y, J Z) \\
& -R(W, Y, W, Z)-R(W, J Y, W, J Z)] .
\end{aligned}
$$

With the help of the equation (1.8) it follows

$$
\bar{R}(W, X, Y, Z)=\bar{R}(Y, Z, W, X)=-\bar{R}(X, W, Y, Z)=-\bar{R}(W, X, Z, Y) .
$$

Using $\bar{\nabla} J=0$ and $\bar{\nabla} g=0$ we obtain

$$
\begin{aligned}
\bar{R}(W, X, Y, Z) & =\bar{R}(W, X, J Y, J Z) \\
& =\bar{R}(J W, J X, Y, Z)=\bar{R}(J W, J X, J Y, J Z) .
\end{aligned}
$$

The general form of the first Bianchi identity (cf. chapter III of [16]) for a connection with torsion yields in the case of parallel torsion:

$$
\underset{X Y Z}{\sigma} \bar{R}(W, X, Y, Z)=-{ }_{X Y Z}^{\sigma} g\left(\left(\nabla_{W} J\right) X,\left(\nabla_{Y} J\right) Z\right) .
$$

In a similar way we get from the second Bianchi identity (cf. chapter III of [16]) for a connection with parallel torsion or from the second Bianchi identity for $\nabla$

$$
-\underset{V W X}{\sigma} \bar{\nabla}_{V}(\bar{R})(W, X, Y, Z)=\underset{V W X}{\sigma} \bar{R}\left(\left(\nabla_{V} J\right) J W, X, Y, Z\right) .
$$

From deriving equation (1.8) and the second Bianchi identity of $\nabla$ one gets after a direct computation

$$
\underset{V W X}{\sigma} \nabla_{V}(\bar{R})(W, X, Y, Z)=\frac{1}{2} g\left(\left(\nabla_{Y}\right) Z, \underset{V W X}{\sigma}\left(\nabla_{X} J\right)\left(\nabla_{V} J\right) J W\right)
$$

which implies

$$
\underset{V W X}{\sigma} \nabla_{V}(\bar{R})(W, X, Y, J Y)=0
$$


Proposition 1.3. The tensor $r$ on a nearly pseudo-Kähler manifold $(M, J, g)$ is parallel with respect to the canonical connection $\bar{\nabla}$.

Proof. Deriving $g(r X, X)=\sum_{i=1}^{2 n} \epsilon_{i} g\left(\left(\nabla_{e_{i}} J\right) X,\left(\nabla_{e_{i}} J\right) X\right)$ one obtains

$$
\begin{aligned}
& g\left(\left(\nabla_{U} r\right) X, X\right)=2 \sum_{i=1}^{2 n} \epsilon_{i} g\left(\left(\nabla_{U, e_{i}}^{2} J\right) X,\left(\nabla_{e_{i}} J\right) X\right) \\
& \stackrel{\sqrt{1.5}}{=}-\sum_{i=1}^{2 n} \epsilon_{i}\left[g\left(\left(\nabla_{U} J\right) e_{i},\left(\nabla_{X} J\right) J\left(\nabla_{e_{i}} J\right) X\right)\right. \\
& +\quad g\left(\left(\nabla_{U} J\right)\left(\nabla_{e_{i}} J\right) X,\left(\nabla_{e_{i}} J\right) J X\right) \\
& \left.+\quad g\left(\left(\nabla_{U} J\right) X,\left(\nabla_{\left(\nabla_{e_{i}} J\right) X} J\right) J e_{i}\right)\right] .
\end{aligned}
$$

We observe that in the first two terms exchanging $e_{i}$ by $J e_{i}$ gives a minus sign. Hence taking an adapted orthogonal frame $\left\{e_{i}\right\}_{i=1}^{2 n}$ yields:

$$
\begin{aligned}
g\left(\left(\nabla_{U} r\right) X, X\right) & =\sum_{i=1}^{2 n} \epsilon_{i} g\left(\left(\nabla_{U} J\right) X,\left(\nabla_{J e_{i}} J\right)\left(\nabla_{e_{i}} J\right) X\right)=-\sum_{i=1}^{2 n} \epsilon_{i} g\left(\left(\nabla_{U} J\right) X,\left(\nabla_{e_{i}} J\right)\left(\nabla_{e_{i}} J\right) J X\right) \\
& =\sum_{i=1}^{2 n} \epsilon_{i} g\left(\left(\nabla_{e_{i}} J\right)\left(\nabla_{U} J\right) X,\left(\nabla_{e_{i}} J\right) J X\right)=g\left(r\left(\nabla_{U} J\right) X, J X\right) .
\end{aligned}
$$

Polarizing this expression shows using that $r$ is $g$-symmetric the following identity

$$
g\left(\left(\nabla_{U} r\right) X, Y\right)=\frac{1}{2} g\left(r\left(\nabla_{U} J\right) X, J Y\right)+\frac{1}{2} g\left(J X, r\left(\nabla_{U} J\right) Y\right) .
$$

As the difference of $\bar{\nabla}$ and $\nabla$ is $-\frac{1}{2} J \nabla J$ the last equation is exactly $\bar{\nabla} r=0$.

Theorem 1.4. Let $(M, J, g)$ be a nearly pseudo-Kähler manifold and let $W, X$ be vector fields on $M$ then it holds

$$
\sum_{i, j=1}^{2 n} \epsilon_{i} \epsilon_{j} g\left(r e_{i}, e_{j}\right)\left[R\left(W, e_{i}, X, e_{j}\right)-5 R\left(W, e_{i}, J X, J e_{j}\right)\right]=0 .
$$

Proof. Let $\left\{e_{i}\right\}_{i=1}^{2 n}$ be an adapted orthogonal frame field. One observes

$$
\begin{aligned}
\sum_{i=1}^{2 n} \epsilon_{i} \bar{R}\left(W, X, e_{i},\left(\nabla_{V} J\right) e_{i}\right) \stackrel{(1.11)}{=} \frac{1}{2} \sum_{i=1}^{2 n} & \epsilon_{i}\left[\bar{R}\left(W, X, e_{i},\left(\nabla_{V} J\right) e_{i}\right)\right. \\
& \left.-\bar{R}\left(W, X, J e_{i},\left(\nabla_{V} J\right) J e_{i}\right)\right]=0
\end{aligned}
$$

and one gets after derivation of the left hand-side

$$
\sum_{i=1}^{2 n} \epsilon_{i}\left[\nabla_{U}(\bar{R})\left(W, X, e_{i},\left(\nabla_{V} J\right) e_{i}\right)+\bar{R}\left(W, X, e_{i}, \nabla_{U, V}^{2}(J) e_{i}\right)\right]=0 .
$$

Taking the trace in $U, V$ and applying the identity (1.6) yields on the second term

$$
\begin{aligned}
& \sum_{i, k=1}^{2 n} \epsilon_{i} \epsilon_{k}\left[\bar{R}\left(W, X, e_{i}, \nabla_{e_{k}, e_{k}}^{2}(J) e_{i}\right)\right]=\sum_{i=1}^{2 n} \epsilon_{i} \bar{R}\left(W, X, e_{i},-r\left(J e_{i}\right)\right) \\
= & -\sum_{i, j=1}^{2 n} \epsilon_{i} \epsilon_{j} g\left(r e_{i}, e_{j}\right) \bar{R}\left(W, X, e_{i}, J e_{j}\right) .
\end{aligned}
$$

From (1.9) we obtain

$$
4 \bar{R}\left(W, J W, e_{i}, J e_{j}\right)=5 R\left(W, J W, e_{i}, J e_{j}\right)-R\left(W, e_{i}, W, e_{j}\right)-R\left(W, J e_{i}, W, J e_{j}\right) .
$$


The first Bianchi identity using an adapted frame implies for $i, j \in\{1, \ldots, n\}$ :

$$
\begin{aligned}
R\left(W, J W, e_{i}, J e_{j}\right) & =-R\left(J W, e_{i}, W, J e_{j}\right)-R\left(e_{i}, W, J W, J e_{j}\right) \\
& =-R\left(W, J e_{j}, J W, e_{i}\right)+R\left(W, e_{i}, J W, J e_{j}\right) \\
& =R\left(W, e_{j+n}, J W, J e_{i+n}\right)+R\left(W, e_{i}, J W, J e_{j}\right) .
\end{aligned}
$$

In an adapted frame it is $g\left(r e_{i+n}, e_{j+n}\right)=g\left(r e_{i}, e_{j}\right)$ with $i, j \in\{1, \ldots, n\}$. Therefore taking the trace in an adapted frame and polarizing yields the claimed identity if we show the vanishing of the trace on the first term of (1.17).

$$
\begin{aligned}
\sum_{i, j=1}^{2 n} \epsilon_{i} \epsilon_{j} \nabla_{e_{j}}(\bar{R})\left(W, J W, e_{i},\left(\nabla_{e_{j}} J\right) e_{i}\right) & =\sum_{i, j, k=1}^{2 n} \epsilon_{i} \epsilon_{j} \epsilon_{k} g\left(\left(\nabla_{e_{j}} J\right) e_{i}, e_{k}\right) \nabla_{e_{j}}(\bar{R})\left(W, J W, e_{i}, e_{k}\right) \\
& =\frac{1}{3} \sum_{i, j, k=1}^{2 n} \underset{i j k}{\sigma}\left[\epsilon_{i} \epsilon_{j} \epsilon_{k} g\left(\left(\nabla_{e_{j}} J\right) e_{i}, e_{k}\right) \nabla_{e_{j}}(\bar{R})\left(W, J W, e_{i}, e_{k}\right)\right]
\end{aligned}
$$

Since $\epsilon_{i} \epsilon_{j} \epsilon_{k} g\left(\left(\nabla_{e_{j}} J\right) e_{i}, e_{k}\right)$ is constant under cyclic permutation of $i, j, k$, the last expression vanishes as a consequence of the curvature identities (1.10) and the identity (1.15). Polarization in $W$ finishes the proof.

\section{First structure Results}

2.1. Small dimensions. For a nearly pseudo-Kähler manifold $\nabla \omega$ is a differential form of type $(3,0)+(0,3)$. In consequence real two- or four-dimensional nearly pseudo-Kähler manifolds are automatically pseudo-Kähler. Six dimensional nearly pseudo-Kähler manifolds are either pseudoKähler manifolds or strict nearly pseudo-Kähler manifolds. In the strict case a nearly pseudoKähler manifold $\left(M^{6}, J, g\right)$ is of constant type, i.e. it holds

$$
g\left(\left(\nabla_{X} J\right) Y,\left(\nabla_{X} J\right) Y\right)=\alpha\left(g(X, X) g(Y, Y)-g(X, Y)^{2}-g(J X, Y)^{2}\right) .
$$

The sign of the type constant $\alpha$ depends on the signature $(p, q)$ with $p+q=6$. In fact it is $\operatorname{sign}(\alpha)=\operatorname{sign}(p-q)$, see section 7 of [14].

2.2. Linear algebra of three-forms. In the following section we consider a (finite dimensional) pseudo-hermitian vector space $(V, J,\langle\cdot, \cdot\rangle)$. Let $\eta \in \Lambda^{3} V^{*}$ be a three-form. We define the support of $\eta$ by

$$
\left.\left.\Sigma_{\eta}=\operatorname{span}\{X\lrcorner Y\right\lrcorner \eta \mid X, Y \in V\right\} \subset V
$$

where we identified $V$ and $V^{*}$ by means of $\langle\cdot, \cdot\rangle$. The name support is motivated by the observation, that for a given $\eta \in \Lambda^{3} V^{*}$ it already holds $\eta \in \Lambda^{3} \Sigma_{\eta}^{*}$, compare Lemma 7 of $[8]$.

In the present paper we are essentially interested in the three-form $g_{p}\left(\left(\nabla_{X} J\right)_{p} Y, Z\right)$ for $X, Y, Z \in$ $T_{p} M$ on a nearly pseudo-Kähler manifold $(M, J, g)$. This three-form is a real form of type $(3,0)+$ $(0,3)$. The type condition implies that $\Sigma_{\eta}$ is a $J$-invariant subspace. In particular it follows that the complex dimension of the support of a non-zero such form is at least three.

In [8] the classification of Levi-Civita flat nearly Kähler manifolds was related to the existence of real three-forms of type $(3,0)+(0,3)$ with isotropic support, i.e. such that $\Sigma_{\eta}$ is an isotropic subspace.

We define the kernel of a three-form $\eta \in \Lambda^{3} V^{*}$ by $\left.\mathcal{K}=\mathcal{K}_{\eta}=\operatorname{ker}(X \mapsto X\lrcorner \eta\right)$.

Lemma 2.1. One has $\mathcal{K}=\Sigma_{\eta}^{\perp}$ and $\Sigma_{\eta}=\mathcal{K}^{\perp}$.

Proof. Suppose, that $X$ is in $\mathcal{K}_{\eta}$, i.e. $\left.X\right\lrcorner \eta=0$. By definition $\Sigma_{\eta}$ is spanned by vectors $U$ satisfying $\langle U, \cdot\rangle=\eta(Y, Z, \cdot)$ for $Y, Z \in V$. This implies $\langle U, X\rangle=\eta(Y, Z, X)=0$, since $\eta$ is a three-form. If $X$ is perpendicular to $\Sigma_{\eta}$ the claim follows from the last equation and $\eta \in \Lambda^{3} \Sigma_{\eta}^{*}$. This means $X$ is in $\mathcal{K}_{\eta}$ if and only if $X$ is perpendicular to $\Sigma_{\eta}$. It follows $\Sigma_{\eta}=\mathcal{K}^{\perp}$.

Lemma 2.2. Let $(V, J,\langle\cdot, \cdot\rangle)$ be a pseudo-hermitian vector space with $\operatorname{dim}_{\mathbb{R}}(V)=8$ then a real three-form $\eta$ of type $(3,0)+(0,3)$ and of non-vanishing length has a (complex) one dimensional kernel $\mathcal{K}_{\eta}$, which admits an orthogonal complement $\left(\mathcal{K}_{\eta}\right)^{\perp}$. Moreover one has $\Sigma_{\eta}=\left(\mathcal{K}_{\eta}\right)^{\perp}$. 
Proof. Let us identify $V$ and $V^{1,0}$. Denote by $\left\{\alpha_{i}\right\}_{i=1}^{4}$ a unitary basis of $\left(V^{1,0}\right)^{*}$ and define a $(4,0)$-form $v$ by $v=\alpha_{1} \wedge \alpha_{2} \wedge \alpha_{3} \wedge \alpha_{4}$. The map

$$
\left.\Phi: V^{1,0} \rightarrow \Lambda^{3}\left(V^{1,0}\right)^{*}, \quad \zeta \mapsto \zeta\right\lrcorner v
$$

yields an isometry. Therefore the (3,0)-form $\rho=\eta+i J^{*} \eta$ is given by $\left.\rho=Z\right\lrcorner v$ for some $Z \in V^{1,0}$ and consequently it follows $Z\lrcorner \rho=0$. As $\Phi$ is an isometry and $\rho$ has non-zero length, we conclude that $Z$ is not isotropic. Denote by $\mathcal{L} \subset \mathcal{K}_{\rho}$ the complex line spanned by $Z$ and by $\mathcal{L}^{\perp} \supset \mathcal{K}_{\rho}^{\perp}$ its orthogonal complement. It remains to prove $\mathcal{L}=\mathcal{K}$ : On the one hand we have $\Sigma_{\rho}=\mathcal{K}_{\rho}^{\perp} \subset \mathcal{L}^{\perp}$ and on the other hand from $\rho \neq 0$ we get $\operatorname{dim}_{\mathbb{C}} \Sigma_{\rho} \geq 3=\operatorname{dim}_{\mathbb{C}} \mathcal{L}^{\perp}$. This shows $\Sigma_{\rho}=\mathcal{L}^{\perp}$ and $\mathcal{K}_{\rho}=\mathcal{L}$.

Remark 2.3. As the reader observes, if $\eta$ has length zero, one can replace the orthogonal complement by the null-space and obtain an analogous statement as in the last proposition.

Lemma 2.4. Let $(V, J,\langle\cdot, \cdot\rangle)$ be a pseudo-hermitian vector space with $\operatorname{dim}_{\mathbb{R}}(V)=10$ then a real three-form $\eta$ of type $(3,0)+(0,3)$ and of non-vanishing length is of the following possible types:

(i) There exists an orthonormal real basis $\left\{f_{i}\right\}_{i=1}^{10}=\left\{e_{1}, J e_{1}, \ldots, e_{5}, J e_{5}\right\}$ and real numbers $\alpha, \beta$ such that

$$
\eta\left(e_{1}, e_{2}, e_{3}\right)=\alpha \neq 0 ; \quad \eta\left(e_{4}, e_{5}, e_{1}\right)=\beta
$$

and $\eta\left(f_{i}, f_{j}, f_{k}\right)=0$ for the cases which are not obtained from (2.3) by skew-symmetry and type relations.

(ii) There exists an orthonormal real basis $\left\{f_{i}\right\}_{i=1}^{10}=\left\{e_{1}, J e_{1}, \ldots, e_{5}, J e_{5}\right\}$ and real numbers $\alpha, \beta$ such that

$$
\eta\left(e_{1}, e_{2}, e_{3}\right)=\alpha \neq 0 ; \quad \eta\left(e_{4}, e_{5}, e_{1}+e_{3}\right)=\beta \text { with }\left\langle e_{1}, e_{1}\right\rangle=-\left\langle e_{3}, e_{3}\right\rangle
$$

and $\eta\left(f_{i}, f_{j}, f_{k}\right)=0$ for the cases which are not obtained from (2.4) by skew-symmetry and type relations.

Proof. Denote by $\left\{\alpha_{i}\right\}_{i=1}^{5}$ a unitary basis of $\left(V^{1,0}\right)^{*}$ and define a $(5,0)$-form $v$ by $v=\alpha_{1} \wedge \alpha_{2} \wedge$ $\alpha_{3} \wedge \alpha_{4} \wedge \alpha_{5}$. The map

$$
\left.\Phi: \Lambda^{2} V^{1,0} \rightarrow \Lambda^{3}\left(V^{1,0}\right)^{*}, \quad \varphi \mapsto \varphi\right\lrcorner v
$$

yields an isometry. Therefore the (3,0)-form $\rho=\eta+i J^{*} \eta$ is given by $\left.\rho=\varphi\right\lrcorner v$ for some $\varphi \in \Lambda^{2} V^{1,0}$. As $\Phi$ is an isometry and $\eta$ has non-zero length, we conclude that $\varphi$ is not isotropic. Define $Z \in\left(V^{1,0}\right)^{*}$ by $\left.\left.\left.Z=\varphi\right\lrcorner \rho=\varphi\right\lrcorner(\varphi\lrcorner \rho\right)$. From $\langle Z, Z\rangle=\langle\varphi, \varphi\rangle^{2}\langle\rho, \rho\rangle$ we obtain that $Z$ is not isotropic. Choosing a unitary basis $\left\{\frac{1}{\sqrt{2}}\left(e_{1}-i J e_{1}\right), \frac{1}{\sqrt{2}}\left(e_{2}-i J e_{2}\right)\right\}$ of the plane $\varphi$ and $Z=\alpha^{\prime} \frac{1}{\sqrt{2}}\left(e_{3}-i J e_{3}\right)^{*}$ for a unit vector $e_{3} \in V$ and $\alpha^{\prime} \in \mathbb{R}-\{0\}$ we consider $\mathcal{B}^{1,0}:=\left\{\frac{1}{\sqrt{2}}\left(e_{1}-i J e_{1}\right), \frac{1}{\sqrt{2}}\left(e_{2}-i J e_{2}\right), \frac{1}{\sqrt{2}}\left(e_{3}-\right.\right.$ $\left.\left.i J e_{3}\right)\right\}$.

Claim: For $\zeta, \chi \in \operatorname{span}_{\mathbb{C}} \mathcal{B}^{1,0}$ it follows $\rho(\zeta, \chi, \cdot) \in \operatorname{span}_{\mathbb{C}}\left(\mathcal{B}^{1,0}\right)^{*}$.

Let us define the map $\tilde{\Phi}: \Lambda^{2}\left(\operatorname{span}_{\mathbb{C}} \mathcal{B}^{1,0}\right) \rightarrow\left(V^{1,0}\right)^{*}$, by linear extension of $\zeta \wedge \chi \mapsto \rho(\zeta, \chi, \cdot)$.

We observe that $\Lambda^{2}\left(\operatorname{span}_{\mathbb{C}} \mathcal{B}^{1,0}\right)$ is a vector space of complex dimension three, which implies that $\operatorname{dim}_{\mathbb{C}}(\operatorname{im} \tilde{\Phi}) \leq 3$. As $\rho$ is a three-form one easily sees that the duals of $e_{j}-i J e_{j}$ for $j=1,2,3$ are contained in im $\tilde{\Phi}$. By the bound on the dimension of im $\tilde{\Phi}$ the components orthogonal to $\left(\operatorname{span} \mathcal{B}^{1,0}\right)^{*}$ vanish. This proves the claim.

Let $W$ be the orthogonal complement of $\operatorname{span}_{\mathbb{C}} \mathcal{B}^{1,0}$. Choose an orthogonal basis $\left\{e_{4}-i J e_{4}, e_{5}-\right.$ $\left.i J e_{5}\right\}$ of $W$. Using that $\rho$ is skew-symmetric we conclude that $\tilde{Z}=\rho\left(e_{4}-i J e_{4}, e_{5}-i J e_{5}, \cdot\right)$ is perpendicular to the dual $W^{*}$ of $W$ and hence an element $\tilde{Z}$ of $\left(\operatorname{span}_{\mathbb{C}} \mathcal{B}^{1,0}\right)^{*}$. If $\langle\tilde{Z}, \tilde{Z}\rangle \neq 0$ we can adapt the basis of $\mathcal{B}^{1,0}$ such that $\tilde{Z}=\beta^{\prime} \frac{1}{\sqrt{2}}\left(e_{1}-i J e_{1}\right)^{*}$. If $\langle\tilde{Z}, \tilde{Z}\rangle=0$ we can achieve $\tilde{Z}=\beta^{\prime} \frac{1}{\sqrt{2}}\left[\left(e_{1}-i J e_{1}\right)+\left(e_{3}-i J e_{3}\right)\right]$ with $\left\langle e_{1}, e_{1}\right\rangle=-\left\langle e_{3}, e_{3}\right\rangle$. Passing to the real basis yields some new constants $\alpha, \beta$ and the claim of the Lemma. 
2.3. Kähler factors and the structure in dimension 8. The aim of this subsection is to split off the pseudo-Kähler factor of a nearly pseudo-Kähler manifold. This will be done by means of the kernel of $\nabla J$ and allows to reduce the (real) dimension from eight to six.

For $p \in M$ we set

$$
\mathcal{K}_{p}=\operatorname{ker}\left(X \in T_{p} M \mapsto \nabla_{X} J\right) .
$$

Theorem 2.5. $\quad$ Let $(M, J, g)$ be a nearly pseudo-Kähler manifold. Suppose, that the distribution $\mathcal{K}$ has constant dimension and admits an orthogonal complement,

(i) then $M$ is locally a pseudo-Riemannian product $M=K \times M_{1}$ of a pseudo-Kähler manifold $K$ and a strict nearly pseudo-Kähler manifold $M_{1}$.

(ii) if $M$ is complete and simply connected then it is a pseudo-Riemannian product $M=$ $K \times M_{1}$ of a pseudo-Kähler manifold $K$ and a strict nearly pseudo-Kähler manifold $M_{1}$.

Proof. The distribution $\mathcal{K}$ is parallel for the canonical connection $\bar{\nabla}$, since $\nabla J$ is $\bar{\nabla}$-parallel. By the formula (1.7) and the nearly Kähler condition it follows $\bar{\nabla}_{X} K=\nabla_{X} K$ for sections $K$ in $\mathcal{K}$ and $X$ in $T M$. This implies that $\mathcal{K}$ is parallel for the Levi-Civita connection and in consequence its orthogonal complement $(\mathcal{K})^{\perp}$ is Levi-Civita parallel. The proof of (i) finishes by the local version of the theorem of de Rham and the proof of (ii) by the global version.

Remark 2.6. There exist nearly pseudo-Kähler manifolds $(M, J, g)$ without pseudo-Kähler de Rham factor, such that $\mathcal{K}_{\eta} \neq\{0\}$ admits no orthogonal complement. In fact there are Levi-Civita flat nearly pseudo-Kähler manifolds constructed in Theorem 1 and 3 of [8] such that the threeform $\eta_{p}(X, Y, Z)=g_{p}\left(J\left(\nabla_{X} J\right) Y, Z\right)$, for $p \in M$, has a support $\Sigma_{\eta} \subset T_{p} M$ which is a maximally isotropic subspace (Here we identified $T_{p} M$ and $T_{p}^{*} M$ via the metric $g$.). Obviously, $J\left(\nabla_{X} J\right) Y$ and $J\left(\nabla_{U} J\right) V$ are elements of the support of $\eta$ for arbitrary $X, Y, U, V \in T_{p} M$. It then follows $0=g\left(J\left(\nabla_{X} J\right) Y, J\left(\nabla_{U} J\right) V\right)=g\left(J\left(\nabla_{J\left(\nabla_{X} J\right) Y} J\right) U, V\right)$ for all $V \in T_{p} M$. Hence it is $\Sigma_{\eta} \subset \mathcal{K}_{\eta}$. Moreover for general reasons we have shown before $\Sigma_{\eta}=\mathcal{K}_{\eta}^{\perp}$ which shows $\mathcal{K}_{\eta} \cap \mathcal{K}_{\eta}^{\perp} \neq\{0\}$ for the above examples. From these examples we learn, that the Theorem 2.5] does not hold true, if there is no orthogonal complement.

Definition 2.7. A nearly pseudo-Kähler manifold $(M, J, g)$ is called nice if the three-form $g((\nabla . J) \cdot, \cdot)$ has non-zero length in each point $p \in M$.

Theorem 2.8. Let $\left(M^{8}, J, g\right)$ be a complete simply connected eight-dimensional nice nearly pseudo-Kähler manifold. Then $M=M_{1} \times M_{2}$ where $M_{1}$ is a two-dimensional Kähler manifold and $M_{2}$ is a six-dimensional strict nearly pseudo-Kähler manifold.

Proof. Since $(M, J, g)$ is a nice nearly pseudo-Kähler manifold we can use Lemma 2.2 to obtain an orthogonal splitting in the two-dimensional distribution $\mathcal{K}$ and its orthogonal complement, which coincides with $\Sigma_{\eta}$. Therefore we are in the situation of Theorem 2.5 (ii).

\subsection{Einstein condition versus reducible holonomy.}

Theorem 2.9. Let $(M, J, g)$ be a nearly pseudo-Kähler manifold.

(i) Suppose that $r$ has more than one eigenvalue, then the canonical Hermitian connection has reduced holonomy.

(ii) If the tensor field $r$ has exactly one eigenvalue then $M$ is a pseudo-Riemannian Einstein manifold.

Proof. (i) Let $\mu_{i}$ for $i=1, \ldots, l$ be the eigenvalues of $r$. Then the decomposition in the according eigenbundles $\operatorname{Eig}\left(\mu_{i}\right)$ is $\bar{\nabla}$-parallel and hence its holonomy is reducible.

(ii) From the identity of Theorem 1.4 and $r=\mu \mathbb{1}_{T M}$ we obtain

$$
0=\sum_{i=1}^{2 n} \epsilon_{i}\left(R\left(W, e_{i}, X, e_{i}\right)-5 R\left(W, e_{i}, J X, J e_{i}\right)\right)=g\left(\left(R i c-5 R i c^{*}\right) W, X\right),
$$

where we used the Bianchi identity and an adapted frame to obtain the last equality. This shows comparing with $r=R i c-R i c^{*}$ that it holds $R i c=\frac{5}{4} \mu$. 
Let us recall, that in the pseudo-Riemannian setting the decomposition into the eigenbundles is not automatically ensured to be an orthogonal direct decomposition. Therefore we introduce the following notion:

Definition 2.10. A nearly pseudo-Kähler manifold $(M, J, g)$ is called decomposable if the above decomposition into the eigenbundles of the tensor $r$ is orthogonal.

Theorem 2.11. A strict nearly pseudo-Kähler six-manifold $\left(M^{6}, J, g\right)$ of constant type $\alpha$ is a pseudo-Riemannian Einstein manifold with Einstein constant $5 \alpha$.

Proof. In an adapted basis we obtain from the symmetries of $\nabla J$

$$
g(r X, X)=2 \sum_{i=1}^{3} \epsilon_{i} g\left(\left(\nabla_{X} J\right) e_{i},\left(\nabla_{X} J\right) e_{i}\right)=-2 \sum_{i=1}^{3} \epsilon_{i} g\left(\left(\nabla_{X} J\right)^{2} e_{i}, e_{i}\right) .
$$

This is exactly minus the trace of the operator $\left(\nabla_{X} J\right)^{2}$ which has a simple form in a cyclic frame. It follows after polarizing $g(r X, Y)=4 \alpha g(X, Y)$. From Theorem 2.9 we compute the Einstein constant $5 \alpha$ where $\alpha$ is the type constant of the strict nearly pseudo-Kähler manifold $M^{6}$.

Proposition 2.12. Let $\left(M^{10}, J, g\right)$ be a nice nearly pseudo-Kähler ten-manifold.

(i) Then the tensor $r$ in a frame of the first type in Lemma 2.4 is given by

$$
\begin{aligned}
r e_{1} & =4\left(\alpha^{2}+\beta^{2}\right) e_{1}, \\
r e_{2} & =4 \alpha^{2} e_{2}, \quad r e_{3}=4 \alpha^{2} e_{3}, \\
r e_{4} & =4 \beta^{2} e_{4}, \quad r e_{5}=4 \beta^{2} e_{5}, \\
r\left(J e_{i}\right) & =J r\left(e_{i}\right), \quad i=1, \ldots, 5,
\end{aligned}
$$

where $\alpha, \beta$ are constants.

(ii) For a frame of the second type in Lemma 2.4 the tensor $r$ is given by

$$
\begin{gathered}
r\left[\begin{array}{l}
e_{1} \\
e_{2} \\
e_{3}
\end{array}\right]=4\left[\begin{array}{ccc}
\alpha^{2}+\beta^{2} \epsilon_{4} \epsilon_{5} & 0 & \beta^{2} \epsilon_{4} \epsilon_{5} \\
0 & \alpha^{2} & 0 \\
\beta^{2} \epsilon_{4} \epsilon_{5} & 0 & \alpha^{2}+\beta^{2} \epsilon_{4} \epsilon_{5}
\end{array}\right]\left[\begin{array}{l}
e_{1} \\
e_{2} \\
e_{3}
\end{array}\right] \\
r e_{4}=0, \\
r e_{5}=4 \beta^{2}\left(2 \epsilon_{1} \epsilon_{4}-1\right) e_{5} \\
r\left(J e_{i}\right)=J r\left(e_{i}\right), \quad i=1, \ldots, 5 .
\end{gathered}
$$

The eigenvalues are $\left\{0 ; 4 \alpha^{2} ; 4 \beta^{2}\left(2 \epsilon_{1} \epsilon_{4}-1\right) ; 4\left(\alpha^{2}+2 \beta^{2} \epsilon_{4} \epsilon_{5}\right)\right\}$, where the eigenbundles are given as

$$
\begin{aligned}
\operatorname{Ker}(r) & =\operatorname{span}\left\{e_{4}, J e_{4}\right\}, \\
\operatorname{Eig}\left(r, 4 \alpha^{2}\right) & =\operatorname{span}\left\{-e_{1}+e_{3}, e_{2},-J e_{1}+J e_{3}, J e_{2}\right\}, \\
\operatorname{Eig}\left(r, 4 \beta^{2}\left(2 \epsilon_{1} \epsilon_{4}-1\right)\right) & =\operatorname{span}\left\{e_{5}, J e_{5}\right\} \\
\left.\operatorname{Eig}\left(r, 4\left(\alpha^{2}+2 \beta^{2} \epsilon_{4} \epsilon_{5}\right)\right)\right) & =\operatorname{span}\left\{e_{1}+e_{3}, J e_{1}+J e_{3}\right\},
\end{aligned}
$$

where $\alpha, \beta$ are constants. For $\beta^{2} \neq 0$ the second case is not decomposable.

(iii) Suppose $\beta=0$ in the case (i) and (ii). Then it follows

$$
\begin{aligned}
\operatorname{Eig}\left(r, 4 \alpha^{2}\right) & =\operatorname{span}\left\{e_{1}, e_{2}, e_{3}, J e_{1}, J e_{2}, J e_{3}\right\} \\
\operatorname{Ker}(r) & =\operatorname{span}\left\{e_{4}, e_{5}, J e_{4}, J e_{5}\right\}
\end{aligned}
$$

Proof. In an adapted basis we obtain from the symmetries of $\nabla J$

$$
g(r X, Y)=2 \sum_{i=1}^{5} \epsilon_{i} g\left(\left(\nabla_{X} J\right) e_{i},\left(\nabla_{Y} J\right) e_{i}\right)=-2 \sum_{i=1}^{5} \epsilon_{i} g\left(\left(\nabla_{Y} J\right)\left(\nabla_{X} J\right) e_{i}, e_{i}\right) .
$$

This is exactly minus the trace of the operator $\left(\nabla_{Y} J\right)\left(\nabla_{X} J\right)$. Using the form of Lemma 2.4 one can calculate $r$ by hand or using computer algebra systems to obtain the claimed results. 
Theorem 2.13. Let $\left(M^{10}, J, g\right)$ be a complete simply connected nice decomposable nearly pseudoKähler manifold of dimension ten. Then $M^{10}$ is of one of the following types

(i) the tensor $r$ has a kernel and $M^{10}=K \times M^{6}$ is a product of a four-dimensional pseudoKähler manifold $K$ and a strict nearly pseudo-Kähler six-manifold $M^{6}$.

(ii) the tensor $r$ has trivial kernel and $r$ has eigenvalues $4\left(\alpha^{2}+\beta^{2}\right)$ with multiplicity $2,4 \alpha^{2}, 4 \beta^{2}$ with multiplicity 4 for some $\alpha, \beta \neq 0$,

A nice nearly pseudo-Kähler manifold $\left(M^{10}, J, g\right)$ is decomposable if the dimension of the kernel of $r$ is not equal to two.

Proof. Since we suppose, that $\left(M^{10}, J, g\right)$ is a nice and decomposable nearly pseudo-Kähler manifold, Proposition 2.12 implies that one has the two different cases:

(i) the distribution $\mathcal{K}$, which is the tangent space of the Kähler factor has dimension four and admits an orthogonal complement of dimension six. This is part (iii) of Proposition 2.12 Part (i) of the Theorem now follows from Theorem 2.5.

(ii) the tensor $r$ has trivial kernel and we are in the situation of Proposition 2.12 part (i) with $\alpha, \beta \neq 0$ and part (ii) follows.

Remark 2.14. Nearly pseudo-Kähler manifolds falling in the second case of the last theorem will be shown to be related to twistor spaces in section 5.5 .

\section{Pseudo-Riemannian submersions}

Let us consider the setting of a pseudo-Riemannian submersion $\pi:(M, g) \rightarrow(N, h)$. The tangent bundle $T M$ of $M$ splits orthogonally into the direct sum

$$
T M=\mathcal{H} \oplus \mathcal{V} .
$$

Denote by $\iota_{\mathcal{H}}, \iota_{\mathcal{V}}$ the canonical inclusions and by $\pi_{\mathcal{H}}, \pi_{\mathcal{V}}$ the canonical projections. We recall the definition [3, 21] of the fundamental tensorial invariants $A$ and $T$ of the submersion $\pi$

$$
\begin{aligned}
& T_{\zeta}=\pi_{\mathcal{H}} \circ \nabla_{\pi_{\mathcal{V}}(\zeta)} \circ \pi_{\mathcal{V}}+\pi_{\mathcal{V}} \circ \nabla_{\pi_{\mathcal{V}}(\zeta)} \circ \pi_{\mathcal{H}}, \\
& A_{\zeta}=\pi_{\mathcal{H}} \circ \nabla_{\pi_{\mathcal{H}}(\zeta)} \circ \pi_{\mathcal{V}}+\pi_{\mathcal{V}} \circ \nabla_{\pi_{\mathcal{H}}(\zeta)} \circ \pi_{\mathcal{H}},
\end{aligned}
$$

where $\zeta$ is a vector field on $M$.

The components of the Levi-Civita connection $\nabla$ are given in the next proposition (compare [21, [3] 9.24 and 9.25).

Proposition 3.1. Let $\pi:(M, g) \rightarrow(N, h)$ be a pseudo-Riemannian submersion, denote by $\nabla$ the Levi-Civita connection of $g$ and define $\nabla^{\mathcal{V}}:=\pi_{\mathcal{V}} \circ \nabla \circ \iota_{\mathcal{V}}$. For vector fields $X, Y$ in $\mathcal{H}$ and $U, V$ in $\mathcal{V}$ we have the following identities

$$
\begin{aligned}
\nabla_{U} V & =\nabla_{U}^{\mathcal{V}} V+T_{U} V \\
\nabla_{U} X & =T_{U} X+\pi_{\mathcal{H}}\left(\nabla_{U} X\right), \\
\nabla_{X} U & =\pi_{\mathcal{V}}\left(\nabla_{X} U\right)+A_{X} U, \\
\nabla_{X} Y & =A_{X} Y+\pi_{\mathcal{H}}\left(\nabla_{X} Y\right), \\
\pi_{\mathcal{V}}[X, Y] & =2 A_{X} Y, \\
g\left(A_{X} Y, U\right) & =-g\left(A_{X} U, Y\right), \text { or more generally } A \text { is alternating. }
\end{aligned}
$$

The canonical variation of the metric $g$ for $t \in \mathbb{R}-\{0\}$ is given by

$$
g_{t}:=\left\{\begin{array}{l}
g(X, Y), \text { for } X, Y \in \mathcal{H}, \\
\operatorname{tg}(V, W), \text { for } V, W \in \mathcal{V}, \\
g(V, X)=0, \text { for } V \in \mathcal{V}, X \in \mathcal{H} .
\end{array}\right.
$$

Lemma 3.2. Denote by $X, Y$ vector fields in $\mathcal{H}$ and by $U, V$ vector fields in $\mathcal{V}$. 
(1) Let $A^{t}$ and $T^{t}$ be the tensorial invariants for $g_{t}$ and $A$ and $T$ those for $g=g_{1}$. Then it holds

$$
\begin{aligned}
& A_{X}^{t} Y=A_{X} Y, \quad A_{X}^{t} U=t A_{X} U \text { and } \\
& T_{U}^{t} V=t T_{U} V, \quad T_{U}^{t} X=T_{U} X
\end{aligned}
$$

(2) $\nabla_{U}^{t} \mathcal{V} V=\nabla_{U}^{\mathcal{V}} V$

(3)

$$
\pi_{\mathcal{H}}\left(\nabla_{X}^{t} Y\right)=\pi_{\mathcal{H}}\left(\nabla_{X} Y\right) \text { and } \pi_{\mathcal{V}}\left(\nabla_{X}^{t} V\right)=\pi_{\mathcal{V}}\left(\nabla_{X} V\right)
$$

$$
\begin{aligned}
& \pi_{\mathcal{H}}\left(\nabla^{t}{ }_{V} X-\nabla_{V} X\right)=(t-1) A_{X} V \\
& \nabla_{U}^{t} V=\nabla_{U} V+(t-1) T_{U} V .
\end{aligned}
$$

Proof. The first part can be found in Lemma 9.69 of [3]. On the right hand-side of the Koszul formulas one only needs the metric $g_{t}$ on $\mathcal{V}$ to determine $\nabla^{t} \mathcal{V}$. This shows $\nabla^{t} \mathcal{V}=\nabla^{\mathcal{V}}$. An analogous argument using the Koszul formulas shows $\pi_{\mathcal{H}}\left(\nabla^{t}{ }_{X} Y\right)=\pi_{\mathcal{H}}\left(\nabla_{X} Y\right)$ and $\pi_{\mathcal{V}}\left(\nabla_{X}^{t} V\right)=\pi_{\mathcal{V}}\left(\nabla_{X} V\right)$. The first part of the point (4) follows from the identities (3.6) and (3.7) and the Koszul formulas. The last equation follows from (1) and (2): $\nabla_{U}^{t} V=\nabla_{U}^{t}{ }_{U}^{\mathcal{V}} V+T_{U}^{t} V=\nabla_{U}^{\mathcal{V}} V+t T_{U} V=\nabla_{U} V+(t-$ 1) $T_{U} V$.

\section{TWISTOR SPACES OVER QUATERNIONIC AND PARA-QUATERNIONIC KÄHLER MANIFOLDS}

In this section we consider pseudo-Riemannian submersions $\pi:(M, g) \rightarrow(N, h)$ endowed with a complex structure $J$ on $M$ which is compatible with the decomposition (3.1).

Lemma 4.1. Let $\pi:(M, g) \rightarrow(N, h)$ be a pseudo-Riemannian submersion endowed with a complex structure $J$ on $M$ which is compatible with the decomposition (3.1). Then $(M, g, J)$ is a pseudo-Kähler manifold if and only if the following equations are satisfied

$$
\begin{aligned}
& \pi_{\mathcal{H}}\left(\left(\nabla_{X} J\right) Y\right)=\pi_{\mathcal{H}}\left(\left(\nabla_{V} J\right) X\right)=0, \\
& \left(\nabla_{U}^{\mathcal{V}} J\right) V=\pi_{\mathcal{V}}\left(\left(\nabla_{X} J\right) V\right)=0, \\
& A_{X}(J Y)-J A_{X} Y=0, \quad A_{X}(J V)-J A_{X} V=0, \\
& T_{V}(J X)-J T_{V} X=0, \quad T_{U}(J V)-J T_{U} V=0,
\end{aligned}
$$

where $X, Y$ are vector fields in $\mathcal{H}$ and $U, V$ are vector fields in $\mathcal{V}$.

Proof. Let $X, Y$ be vector fields in $\mathcal{H}$ and $U, V$ be vector fields in $\mathcal{V}$. Then it follows from comparing components in $\mathcal{H} \oplus \mathcal{V}$

$$
\begin{aligned}
&\left(\nabla_{X} J\right) Y\left.=\pi_{\mathcal{H}}\left(\left(\nabla_{X} J\right) Y\right)+\pi_{\mathcal{V}}\left(\nabla_{X} J\right) Y\right)=\pi_{\mathcal{H}}\left(\left(\nabla_{X} J\right) Y\right)+\left(A_{X}(J Y)-J A_{X} Y\right), \\
&\left(\nabla_{X} J\right) V\left.=\pi_{\mathcal{H}}\left(\left(\nabla_{X} J\right) V\right)+\pi_{\mathcal{V}}\left(\nabla_{X} J\right) V\right)=\pi_{\mathcal{V}}\left(\left(\nabla_{X} J\right) V\right)+\left(A_{X}(J V)-J A_{X} V\right), \\
&\left.\left(\nabla_{V} J\right) X=\pi_{\mathcal{H}}\left(\left(\nabla_{V} J\right) X\right)+\pi_{\mathcal{V}}\left(\nabla_{V} J\right) X\right)=\pi_{\mathcal{H}}\left(\left(\nabla_{V} J\right) X\right)+\left(T_{V}(J X)-J T_{V} X\right), \\
&\left.\left(\nabla_{U} J\right) V=\pi_{\mathcal{H}}\left(\left(\nabla_{U} J\right) V\right)+\pi_{\mathcal{V}}\left(\nabla_{U} J\right) V\right)=\left(\nabla_{U}^{\mathcal{V}} J\right) V+\left(T_{U}(J V)-J T_{U} V\right) .
\end{aligned}
$$

Further we define a second complex structure by

$$
\hat{J}:=\left\{\begin{array}{c}
J \text { on } \mathcal{H} \\
-J \text { on } \mathcal{V} .
\end{array}\right.
$$

We observe that $\hat{\hat{J}}=J$. This construction was made in [19] for the Riemannian setting and imitates the construction on twistor spaces. 
Proposition 4.2. $\quad$ Suppose, that the foliation induced by the pseudo-Riemannian submersion $\pi$ is totally geodesic and that $(M, J, g)$ is a pseudo-Kähler manifold and $J$ is compatible with the decomposition (3.1), then the manifold $\left(M, \hat{g}=g_{\frac{1}{2}}, \hat{J}\right)$ is a nearly pseudo-Kähler manifold. The distributions $\mathcal{H}$ and $\mathcal{V}$ are parallel with respect to the canonical Hermitian connection $\bar{\nabla}$ of $(M, \hat{g}, \hat{J})$. In other words the nearly pseudo-Kähler manifold $(M, \hat{g}, \hat{J})$ has reducible holonomy.

Proof. Let $U, V$ be vector fields in $\mathcal{V}$ and $X, Y$ be vector fields in $\mathcal{H}:$ In the following $\hat{\nabla}$ is the LeviCivita connection of $\hat{g}$. Since the fibers are totally geodesic, i.e. $T \equiv 0$, we obtain from equation (3.2), that $\hat{\nabla}_{U} V=\hat{\nabla}_{U}^{\mathcal{V}} V+\hat{T}_{U} V=\nabla_{U}^{\mathcal{V}} V+T_{U} V=\nabla_{U} V$, which yields $\left(\hat{\nabla}_{U} \hat{J}\right) V=-\left(\nabla_{U} J\right) V=0$. In the sequel we denote the O'Neill tensors of the pseudo-Riemannian foliations induced by $\mathcal{V}$ on $(M, g)$ and on $(M, \hat{g})$ by $A$ and $\hat{A}$, respectively. From Lemma 3.2 it follows $A_{X} Y=\hat{A}_{X} Y$ and consequently the same Lemma yields $\nabla_{X} Y=\hat{\nabla}_{X} Y$.

Since $(M, g)$ is Kähler, Lemma 4.1 implies $A \circ J=J \circ A$ and we compute

$$
\begin{array}{rll}
\left(\hat{\nabla}_{X} \hat{J}\right) Y & = & \hat{\nabla}_{X}(\hat{J} Y)-\hat{J} \hat{\nabla}_{X} Y \\
= & \pi_{\mathcal{H}}\left[\hat{\nabla}_{X}(J Y)\right]+\pi_{\mathcal{V}}\left[\hat{\nabla}_{X}(J Y)\right]-\hat{J}\left(\pi_{\mathcal{H}}\left(\hat{\nabla}_{X} Y\right)+\pi_{\mathcal{V}}\left(\hat{\nabla}_{X} Y\right)\right) \\
= & \pi_{\mathcal{H}}\left[\hat{\nabla}_{X}(J Y)-J \hat{\nabla}_{X} Y\right]+\pi_{\mathcal{V}}\left[\hat{\nabla}_{X}(J Y)+J \hat{\nabla}_{X} Y\right] \\
= & \pi_{\mathcal{H}}\left(\left(\hat{\nabla}_{X} J\right) Y\right)+\hat{A}_{X}(J Y)+J \hat{A}_{X} Y \\
& \pi_{\mathcal{H}}\left(\left(\nabla_{X} J\right) Y\right)+2 A_{X}(J Y) \stackrel{\text { (3.1) }}{=} 2 A_{X}(J Y)=2 J A_{X} Y .
\end{array}
$$

With the identity $A_{X} V=2 \hat{A}_{X} V$ of Lemma 3.2 we get

$$
\begin{aligned}
& \left(\hat{\nabla}_{X} \hat{J}\right) V \quad=\quad \hat{\nabla}_{X}(\hat{J} V)-\hat{J} \hat{\nabla}_{X} V \\
& =\quad-\pi_{\mathcal{V}}\left(\hat{\nabla}_{X}(J V)\right)-\pi_{\mathcal{H}}\left(\hat{\nabla}_{X}(J V)\right)+J \pi_{\mathcal{V}}\left(\hat{\nabla}_{X} V\right)-J \pi_{\mathcal{H}}\left(\hat{\nabla}_{X} V\right) \\
& =\quad-\pi_{\mathcal{V}}\left(\left(\hat{\nabla}_{X} J\right) V\right)-\hat{A}_{X} J V-J \hat{A}_{X} V \\
& \text { [3.8), (3.100, (4.3) }-\pi_{\mathcal{V}}\left(\left(\nabla_{X} J\right) V\right)-J A_{X} V=-A_{X} J V .
\end{aligned}
$$

The vanishing of the second fundamental form $T$, equation (3.9) and a second time $A_{X} V=2 \hat{A}_{X} V$ show

$$
\begin{array}{rll}
\left(\hat{\nabla}_{V} \hat{J}\right) X \stackrel{=}{=} & \pi_{\mathcal{V}}\left(\hat{\nabla}_{V}(J X)\right)+\pi_{\mathcal{V}}\left(J \hat{\nabla}_{V} X\right)+\pi_{\mathcal{H}}\left(\hat{\nabla}_{V}(J X)-J \hat{\nabla}_{V} X\right) \\
\stackrel{\sqrt{3.11}}{=} & \hat{T}_{V}(J X)+J\left(\hat{T}_{V} X\right)+\pi_{\mathcal{H}}\left(\left(\nabla_{V} J\right) X\right)+\frac{1}{2}\left(J A_{X} V-A_{J X} V\right)=J A_{X} V
\end{array}
$$

where we used $A_{J X} V=-J A_{X} V$ which follows, since $A_{X}$ is alternating (compare equation (3.7)) and commutes with $J$. The next Lemma finishes the proof.

Lemma 4.3. 1.) Suppose, that $(M, \hat{J}, \hat{g})$ is a nearly pseudo-Kähler manifold and $\hat{J}$ is compatible with the decomposition (3.1), then the following statements are equivalent:

(i) the splitting (3.1) is $\bar{\nabla}$-parallel,

(ii) the fundamental tensors $\hat{A}$ and $\hat{T}$ satisfy:

$$
\begin{aligned}
& \hat{T}_{V} X=0, \quad \hat{J} \hat{T}_{V} W=-\hat{T}_{V} \hat{J} W \Leftrightarrow \check{J} \hat{T}_{V} W=\hat{T}_{V} \check{J} W \text { for } \check{J}=\hat{\hat{J}}, \\
& \hat{A}_{X} V=\frac{1}{2} \hat{J}\left(\hat{\nabla}_{X} \hat{J}\right) V, \quad \hat{A}_{X} Y=\frac{1}{2} \pi_{\mathcal{V}}\left(\hat{J}\left(\hat{\nabla}_{X} \hat{J}\right) Y\right) .
\end{aligned}
$$

2.) If it holds $\left(\hat{\nabla}_{V} \hat{J}\right) W=0$ then $\bar{\nabla}_{V} W \in \mathcal{V}$ for $V, W \in \mathcal{V}$ is equivalent to $T_{V} W=0$. Moreover it is $(\hat{\nabla} \mathcal{V}, \hat{J}) W=0$.

Proof. First we compute

$$
\begin{aligned}
\bar{\nabla}_{V} W & =\hat{\nabla}_{V} W-\frac{1}{2} \hat{J}\left(\hat{\nabla}_{V} \hat{J}\right) W \\
& =\hat{\nabla}_{V}^{\mathcal{V}} W+\hat{T}_{V} W-\frac{1}{2} \hat{J}\left(\hat{\nabla}_{V}^{\mathcal{V}} \hat{J}\right) W-\frac{1}{2}\left(\hat{J} \hat{T}_{V}(\hat{J} W)+\hat{T}_{V} W\right) \\
& =\hat{\nabla}_{V}^{\mathcal{V}} W-\frac{1}{2} \hat{J}\left(\hat{\nabla}_{V}^{\mathcal{V}} \hat{J}\right) W+\frac{1}{2}\left(\hat{T}_{V} W-\hat{J} \hat{T}_{V}(\hat{J} W)\right)
\end{aligned}
$$


The first two terms lie in $\mathcal{V}$, the second terms lie in $\mathcal{H}$ and therefore the expression is in $\mathcal{V}$ if and only if $\hat{J} \hat{T}_{V}(\hat{J} W)=\hat{T}_{V} W \Leftrightarrow \hat{T}_{V}(\hat{J} W)=-\hat{J} \hat{T}_{V} W$. From $0=\pi_{\mathcal{H}}\left(\bar{\nabla}_{X} V\right)=\pi_{\mathcal{H}}\left(\hat{\nabla}_{X} V-\frac{1}{2} \hat{J}\left(\hat{\nabla}{ }_{X} \hat{J}\right) V\right)$ one determines

$$
\hat{A}_{X} V=\pi_{\mathcal{H}}\left(\hat{\nabla}_{X} V\right)=\frac{1}{2} \pi_{\mathcal{H}}\left(\hat{J}\left(\hat{\nabla}_{X} \hat{J}\right) V\right)=\frac{1}{2} \hat{J}\left(\hat{\nabla}_{X} \hat{J}\right) V
$$

The last equality follows from Lemma 5.3 (ii) part b). Conversely, if $\hat{A}_{X} V$ is given by the last formula one gets

$$
\bar{\nabla}_{X} V=\hat{\nabla}_{X} V-\frac{1}{2} \hat{J}\left(\hat{\nabla}_{X} \hat{J}\right) V=\hat{\nabla}_{X} V-\hat{A}_{X} V=\pi_{\mathcal{V}}\left(\hat{\nabla}_{X} V\right) \in \mathcal{V}
$$

With the same identity we calculate

$$
\pi_{\mathcal{V}}\left(\bar{\nabla}_{V} X\right)=\pi_{\mathcal{V}}\left(\hat{\nabla}_{V} X-\frac{1}{2} \hat{J}\left(\hat{\nabla}_{V} \hat{J}\right) X\right)=\pi_{\mathcal{V}}\left(\hat{\nabla}_{V} X+\hat{A}_{X} V\right)=\pi_{\mathcal{V}}\left(\hat{\nabla}_{V} X\right)
$$

This is in $\mathcal{H}$ if and only if $\hat{T}_{V} X=\pi_{\mathcal{V}}\left(\hat{\nabla}_{V} X\right)=0$. The last component, i.e. $\pi_{\mathcal{V}}\left(\bar{\nabla}_{X} Y\right)=$ $\pi_{\mathcal{V}}\left(\hat{\nabla}_{X} Y-\frac{1}{2} \hat{J}\left(\hat{\nabla}_{X} \hat{J}\right) Y\right)$ is zero if and only if we have $\hat{A}_{X} Y=\pi_{\mathcal{V}}\left(\hat{\nabla}_{X} Y\right)=\frac{1}{2} \pi_{\mathcal{V}}\left(\hat{J}\left(\hat{\nabla}_{X} \hat{J}\right) Y\right)$.

2.) With $\left(\hat{\nabla}_{V} \hat{J}\right) W=0$ we calculate

$$
\bar{\nabla}_{V} W=\hat{\nabla}_{V} W+\frac{1}{2}\left(\hat{\nabla}_{V} \hat{J}\right) \hat{J} W=\hat{\nabla}_{V}^{\mathcal{V}} W+\hat{T}_{V} W
$$

This lies in $\mathcal{V}$ if and only if $\hat{T}_{V} W=0$.

We apply Proposition 4.2 to twistor spaces and obtain.

Corollary 4.4. The twistor space $\mathcal{Z}$ of a quaternionic Kähler manifold of dimension $4 k$ with negative scalar curvature admits a canonical nearly pseudo-Kähler structure of reducible holonomy contained in $U(1) \times U(2 k)$.

Proof. We remark that in negative scalar curvature the twistor space of a quaternionic Kähler manifold is the total space of a pseudo-Riemannian submersion with totally geodesic fibers. It admits a compatible pseudo-Kähler structure of signature $(2,4 k)$, cf. Besse [3] 14.86 b). The assumption of positive scalar curvature is often made to obtain a positive definite metric on $\mathcal{Z}$. Here we focus on pseudo-Riemannian metrics and consequently on negative scalar curvature.

Proposition 4.5. The twistor spaces $\mathcal{Z}$ of non-compact duals of Wolf spaces and of Alekseevskian spaces admit a nearly pseudo-Kähler structure.

Proof. Non-compact duals of Wolf spaces are known [22] to be quaternionic Kähler manifolds of negative scalar curvature. The same holds for Alekseevskian spaces [1, 6].

Studying the lists given in [1, 6, 22] we find examples of six-dimensional nearly pseudo-Kähler manifolds.

Corollary 4.6. The twistor spaces $\mathcal{Z}$ of $\tilde{\mathbb{H}} P^{1}=\operatorname{Sp}(1,1) / \operatorname{Sp}(1) \operatorname{Sp}(1)$ and $S U(1,2) / S(U(1) U(2))$ provide six-dimensional nearly pseudo-Kähler manifolds.

Remark 4.7. The situation in negative scalar curvature is more flexible than in the positive case. This is illustrated by the following results in this area: In the main theorem of [17] it is shown that the moduli space of complete quaternionic Kähler metrics on $\mathbb{R}^{4 n}$ is infinite dimensional. A construction of super-string theory, called the c-map [11, yields continuous families of negatively curved quaternionic Kähler manifolds. These results show that Corollary 4.4 is a good source of examples.

Another source of examples is given by twistor spaces over para-quaternionic Kähler manifolds. Since these manifolds are less classical than quaternionic Kähler manifolds, we recall some definitions (cf. [2] and references therein). 
Definition 4.8. Let $\left(\epsilon_{1}, \epsilon_{2}, \epsilon_{3}\right)=(-1,1,1)$ or some permutation thereof. An almost paraquaternionic structure on a differentiable manifold $M^{4 k}$ is a rank 3 sub-bundle $Q \subset \operatorname{End}(T M)$, which is locally generated by three anti-commuting endomorphism-fields $J_{1}, J_{2}, J_{3}=J_{1} J_{2}$. These satisfy $J_{i}^{2}=\epsilon_{i} I d$ for $i=1, \ldots, 3$. Such a triple is called standard local basis of $Q$. A linear torsionfree connection preserving $Q$ is called para-quaternionic connection. An almost para-quaternionic structure is called a para-quaternionic structure if it admits a para-quaternionic connection. An almost para-quaternionic hermitian structure $(M, Q, g)$ is a pseudo-Riemannian manifold endowed with a para-quaternionic structure such that $Q$ consists of skew-symmetric endomorphisms. For $n>1\left(M^{4 k}, Q, g\right)$ is a para-quaternionic Kähler manifold if $Q$ is preserved by the Levi-Civita connection of $g$. In dimension 4 a para-quaternionic Kähler manifold $M^{4}$ is an anti-self-dual Einstein manifold.

We use the same notions omitting the word "para" for the quaternionic case. The condition that $Q$ is preserved by the Levi-Civita connection is in a given standard local basis $\left\{J_{i}\right\}_{i=1}^{3}$ of $Q$ equivalent to the equations

$$
\nabla_{X} J_{i}=-\theta_{k}(X) \epsilon_{j} J_{j}+\theta_{j}(X) \epsilon_{k} J_{k}, \text { for } X \in T M,
$$

where $i, j, k$ is a cyclic permutation of $1,2,3$ and $\left\{\theta_{i}\right\}_{i=1}^{3}$ are local one-forms. In the context of para-quaternionic manifolds one can define twistor spaces for $s=1,0,-1$

$$
\mathcal{Z}^{s}:=\left\{A \in Q \mid A^{2}=s I d, \text { with } A \neq 0\right\} .
$$

The case of interest in this text is $\mathcal{Z}=\mathcal{Z}^{-1}$, since this twistor space is a complex manifold, such that the conditions of Proposition 4.2 hold true (cf. [2]). Therefore we obtain the following examples of nearly pseudo-Kähler manifolds.

Corollary 4.9. The twistor space $\mathcal{Z}$ of a para-quaternionic Kähler manifold with non-zero scalar curvature of dimension $4 k$ admits a canonical nearly pseudo-Kähler structure of reducible holonomy contained in $U(k, k) \times U(1)$.

Example 4.10. The para-quaternions $\widetilde{\mathbb{H}}$ are the $\mathbb{R}$-algebra generated by $\{1, i, j, k\}$ subject to the relations $i^{2}=-1, j^{2}=k^{2}=1, i j=-j i=k$. Like the quaternions, the para-quaternions are a real Clifford algebra which in the convention of [18] is $\widetilde{\mathbb{H}}=\mathcal{C} l_{1,1} \cong \mathcal{C} l_{0,2} \cong \mathbb{R}(2)$. One defines the para-quaternionic projective space $\widetilde{\mathbb{H}} P^{n}$ by the obvious equivalence relation on the para-quaternionic right-module $\widetilde{\mathbb{H}}^{n+1}$ of $(n+1)$-tuples of para-quaternions. The manifold $\widetilde{\mathbb{H}} P^{n}$ is a para-quaternionic Kähler manifold [4] in analogue to quaternionic projective space $\mathbb{H} P^{n}$. This yields examples of the type described in the last Corollary.

\section{REDUCIBLE NEARLY PSEUdO-KäHLER MANIFOLDS}

In this section we study the case of a nearly pseudo-Kähler manifold $\left(M^{2 n}, J, g\right)$, such that the holonomy of the canonical connection $\bar{\nabla}$ is reducible, in the sense that the tangent bundle $T M$ admits a splitting

$$
T M=\mathcal{H} \oplus \mathcal{V}
$$

into two $\bar{\nabla}$-parallel sub-bundles $\mathcal{H}, \mathcal{V}$, which are orthogonal and invariant with respect to the almost complex structure $J$.

5.1. General properties. In this subsection we carefully check, generalizing 20 to pseudoRiemannian foliations, the information which follows from the decomposition into the $J$-invariant sub-bundles.

Lemma 5.1. In the situation of this section and for a vector field $X$ in $\mathcal{H}$, a vector field $Y$ in $T M$ and vector fields $U, V$ in $\mathcal{V}$ it is

$$
\bar{R}(X, Y, U, V)=g\left(\left[\nabla_{U} J, \nabla_{V} J\right] X, Y\right)-g\left(\left(\nabla_{X} J\right) Y,\left(\nabla_{U} J\right) V\right) .
$$


Proof. Since $\mathcal{H}$ and $\mathcal{V}$ are $\bar{\nabla}$-parallel it follows $\bar{R}(Y, U, X, V)=0$ and using equation (1.8) we get

$$
\begin{aligned}
R(Y, U, X, V) & =\frac{1}{2} g\left(\left(\nabla_{Y} J\right) U,\left(\nabla_{X} J\right) V\right) \\
& -\frac{1}{4}\left[g\left(\left(\nabla_{Y} J\right) X,\left(\nabla_{U} J\right) V\right)-g\left(\left(\nabla_{Y} J\right) V,\left(\nabla_{U} J\right) X\right)\right] \\
& =-\frac{1}{2} g\left(\left(\nabla_{V} J\right)\left(\nabla_{U} J\right) Y, X\right) \\
& -\frac{1}{4}\left[g\left(\left(\nabla_{Y} J\right) X,\left(\nabla_{U} J\right) V\right)-g\left(\left(\nabla_{U} J\right)\left(\nabla_{V} J\right) Y, X\right)\right] .
\end{aligned}
$$

The first Bianchi identity yields

$$
\begin{aligned}
R(X, Y, U, V) & =-R(Y, U, X, V)-R(U, X, Y, V) \\
& =-R(Y, U, X, V)+R(X, U, Y, V) \\
& =\frac{3}{4} g\left(\left[\left(\nabla_{V} J\right),\left(\nabla_{U} J\right)\right] Y, X\right)+\frac{1}{2} g\left(\left(\nabla_{Y} J\right) X,\left(\nabla_{U} J\right) V\right) .
\end{aligned}
$$

Replacing the last expression into

$$
\begin{aligned}
\bar{R}(X, Y, U, V) & =R(X, Y, U, V)-\frac{1}{2} g\left(\left(\nabla_{X} J\right) Y,\left(\nabla_{U} J\right) V\right) \\
& +\frac{1}{4}\left[g\left(\left(\nabla_{X} J\right) U,\left(\nabla_{Y} J\right) V\right)-g\left(\left(\nabla_{X} J\right) V,\left(\nabla_{Y} J\right) U\right)\right]
\end{aligned}
$$

proves the Lemma.

Corollary 5.2. $\quad$ For vector fields $X, Y$ in $\mathcal{H}$ and $V, W$ in $\mathcal{V}$ one has

(i) $\left(\nabla_{X} J\right)\left(\nabla_{V} J\right) W=0 ;\left(\nabla_{V} J\right)\left(\nabla_{X} J\right) Y=0$;

(ii) $\left(\nabla_{X} J\right)\left(\nabla_{Y} J\right) Z$ belongs to $\mathcal{H}$ for all $Z \in \Gamma(\mathcal{H})$;

(iii) $\left(\nabla_{V} J\right)\left(\nabla_{W} J\right) X$ belongs to $\mathcal{H}$; and $\left(\nabla_{X} J\right)\left(\nabla_{Y} J\right) V$ belongs to $\mathcal{V}$.

Proof.

(i) follows from the fact, that $\bar{R}(J X, J Y, V, W)=\bar{R}(X, Y, V, W)$ and that the first term of equation (5.1) has the same symmetry with respect to $J$. This yields on the one hand

$$
g\left(\left(\nabla_{J X} J\right) J Y,\left(\nabla_{V} J\right) W\right)=g\left(\left(\nabla_{X} J\right) Y,\left(\nabla_{V} J\right) W\right)
$$

and on the other hand it is

$$
g\left(\left(\nabla_{J X} J\right) J Y,\left(\nabla_{V} J\right) W\right)=-g\left(\left(\nabla_{X} J\right) Y,\left(\nabla_{V} J\right) W\right) .
$$

Consequently one has $g\left(\left(\nabla_{X} J\right) Y,\left(\nabla_{V} J\right) W\right)=0$. Exchanging $\mathcal{H}$ and $\mathcal{V}$ finishes part (i).

(ii) From (i) one gets the vanishing of

$g\left(\left(\nabla_{V} J\right)\left(\nabla_{Y} J\right) Z, X\right)=g\left(Z,\left(\nabla_{Y} J\right)\left(\nabla_{V} J\right) X\right)=-g\left(Z,\left(\nabla_{Y} J\right)\left(\nabla_{X} J\right) V\right)=-g\left(\left(\nabla_{X} J\right)\left(\nabla_{Y} J\right) Z, V\right)$.

(iii) From (i) it follows $0=\bar{R}(X, U, V, W)=g\left(\left[\nabla_{V} J, \nabla_{W} J\right] X, U\right)$. This yields $\left[\nabla_{V} J, \nabla_{W} J\right] X \in \mathcal{H}$ and by $\left[\nabla_{V} J, \nabla_{J W} J\right] J X=-\left\{\nabla_{V} J, \nabla_{W} J\right\} X \in \mathcal{H}$ we get the first part. The second part follows by replacing $\mathcal{H}$ and $\mathcal{V}$.

5.2. Co-dimension two. Motivated by the above section on twistor spaces we suppose from now on that the real dimension of $\mathcal{V}$ is two.

Lemma 5.3. $\quad$ Let $\operatorname{dim}_{\mathbb{R}}(\mathcal{V})=2$.

(i) Then the restriction of the metric $g$ is either of signature $(2,0)$ or $(0,2)$.

(ii) a) $T(V, W)=0$ for all $V, W \in \mathcal{V}$.

b) $T(X, U) \in \mathcal{H}$ for all $X \in \mathcal{H}$ and $U \in \mathcal{V}$.

c) In dimension six it is $T(X, Y) \in \mathcal{V}$ for all $X, Y \in \mathcal{H}$.

d) $\operatorname{Span}\left\{\pi_{\mathcal{V}}(T(X, Y)) \mid X, Y \in \mathcal{H}\right\}=\mathcal{V}$. 
Proof. Let $V \in \mathcal{V}$ with $g(V, V) \neq 0$, then it is $\mathcal{V}=\operatorname{span}\{V, J V\}$. (i) It holds $g(J V, J V)=g(V, V) \neq$ 0 . This proves (i).

(ii) In the following we denote by $X, Y$ (local) sections of $\mathcal{H}$ and by $U, V$ (local) sections of $\mathcal{V}$ : The part a) follows from $T(V, V)=T(J V, J V)=0$ and from the formula for the torsion $T(V, J V)=-J\left(\nabla_{V} J\right) J V=-\left(\nabla_{V} J\right) V=0$.

Part b) follows from the fact that $g\left(\left(\nabla_{U} J\right) Y, V\right)$ and $g\left(\left(\nabla_{U} J\right) Y, J V\right)$ are three-forms: This implies, that one has $g\left(\left(\nabla_{U} J\right) X, V\right)=-g\left(\left(\nabla_{U} J\right) V, X\right) \stackrel{a)}{=} 0$ and $g\left(\left(\nabla_{U} J\right) X, J V\right)=-g\left(\left(\nabla_{U} J\right) V, J X\right) \stackrel{a)}{=} 0$. Hence the projection of $T(X, U)$ on $\mathcal{V}$ vanishes and part b) follows. Next we show part d). There exists a pair $X, Y$, such that $\pi_{\mathcal{V}}(T(X, Y)) \neq 0$. Then it follows $\pi_{\mathcal{V}}(T(X, J Y))=-J \pi_{\mathcal{V}}(T(X, Y)) \neq$ 0 and part d) holds true. If there were no such pair $X, Y$ then it follows $-g(T(X, Y), V)=$ $g\left(J\left(\nabla_{X} J\right) Y, V\right)=-g\left(J\left(\nabla_{V} J\right) Y, X\right)=0$ for all $X, Y \in \mathcal{H}, V \in \mathcal{V}$ and consequently using a) and b) it follows $\nabla_{V} J=0$. This contradicts the fact, that $M$ is strict nearly Kähler. It remains part c). As $T(X, Y) \neq 0$ it follows that $X$ and $Y$ are linear independent. From the symmetries of $T(X, Y)$ one concludes, that $\operatorname{span}_{\mathbb{R}}\{X, Y, J X, J Y\} \subset \mathcal{H}$ has real dimension 4 and hence coincides with $\mathcal{H}$. Using the symmetries of $\nabla J$ one gets $T(X, Y), T(X, J Y), T(J X, Y), T(J X, J Y) \in \mathcal{V}$. This finishes the proof.

Corollary 5.4. Let $\operatorname{dim}_{\mathbb{R}}(\mathcal{V})=2$. Then the foliation $\mathcal{V}$ has totally geodesic fibers and the O'Neill tensor is given by $A_{X} Y=\frac{1}{2} \pi_{\mathcal{V}}\left(J\left(\nabla_{X} J\right) Y\right)$ and $A_{X} V=\frac{1}{2} J\left(\nabla_{X} J\right) V$. Moreover it is $\nabla^{\mathcal{V}} J=0$.

Proof. From Lemma 5.3 (ii) a) we obtain $\left(\nabla_{V} J\right) W=0$ with $V, W \in \Gamma(\mathcal{V})$. By Lemma 4.3 part 2) it follows $T_{V} W=0$ and $\nabla^{\mathcal{V}} J=0$, since the decomposition $\mathcal{H} \oplus \mathcal{V}$ is $\bar{\nabla}$ parallel. Part 1) of Lemma 4.3 finishes the proof.

Proposition 5.5. Let $(M, J, g)$ be a nearly pseudo-Kähler manifold such that the property of Lemma 5.3 (ii) c) is satisfied and such that $\mathcal{V}$ has dimension 2 , then $\left(M, \breve{J}=\hat{J}, \check{g}=g_{2}\right)$ is a pseudo-Kähler manifold].

It is natural to suppose the property of Lemma 5.3 (ii) c), since this holds true in the cases of twistorial type which are studied in the next sections.

Proof. By the last Corollary the data of the submersion is $\check{T}=T \equiv 0, A_{X} Y=\check{A}_{X} Y=$ $\frac{1}{2} \pi_{\mathcal{V}}\left(J\left(\nabla_{X} J\right) Y\right)$ and $\check{A}_{X} V=2 A_{X} V=J\left(\nabla_{X} J\right) V$. Since $A$ anti-commutes with $J$ it commutes with $\check{J}$. This yields the conditions (4.3) and (4.4) of Lemma 4.1 on the triple $\check{A}, \check{T}, \check{J}$. Further it holds $\nabla^{\mathcal{V}} J=0$. From the reasoning of equation (4.5) we obtain $\pi_{\mathcal{H}}\left(\left(\check{\nabla}_{X} \check{J}\right) Y\right)=\pi_{\mathcal{H}}\left(\left(\nabla_{X} J\right) Y\right)$ which vanishes by the property of Lemma 5.3 (ii) c). By an analogous argument we get from equation (4.6) the identity $\pi_{\mathcal{V}}\left(\left(\check{\nabla}_{X} \breve{J}\right) V\right)=-\pi_{\mathcal{V}}\left(\left(\nabla_{X} J\right) V\right)$. This vanishes by Lemma 5.3 (ii) b). From equation (4.7) we derive $-\pi_{\mathcal{H}}\left(\left(\nabla_{X} J\right) V\right) \stackrel{n . K .}{=} \pi_{\mathcal{H}}\left(\left(\nabla_{V} J\right) X\right)=\pi_{\mathcal{H}}\left(\left(\check{\nabla}_{V} \breve{J}\right) X\right)+2 \pi_{\mathcal{H}}\left(J A_{X} V\right)$. The definition of $A_{X} V$ yields $\pi_{\mathcal{H}}\left(\left(\check{\nabla}_{V} \check{J}\right) X\right)=0$. These are all the identities needed to apply Lemma 4.1

Proposition 5.6. Let $X, Y$ be vector fields in $\mathcal{H}$ and $V_{1}, V_{2}, V_{3}$ be vector fields in $\mathcal{V}$. Suppose that it holds $T(V, W)=0$ for all $V, W \in \mathcal{V}$ then it is

$$
\bar{R}\left(\left(\nabla_{X} J\right) J Y, V_{1}, V_{2}, V_{3}\right)=g\left(J Y,\left[\nabla_{V_{1}} J,\left[\nabla_{V_{2}} J, \nabla_{V_{3}} J\right]\right] X\right) .
$$

Moreover, one has $\bar{\nabla}_{U} \bar{R}\left(V_{1}, V_{2}, V_{3}, V_{4}\right)=0$.

Proof. For $V_{1}, V_{2}, V_{3} \in \mathcal{V}$ and $X \in \mathcal{H}$ the second Bianchi identity gives

$$
-\underset{X Y V_{1}}{\sigma} \bar{\nabla}_{X}(\bar{R})\left(Y, V_{1}, V_{2}, V_{3}\right)=\underset{X Y V_{1}}{\sigma} \bar{R}\left(\left(\nabla_{X} J\right) J Y, V_{1}, V_{2}, V_{3}\right) .
$$

As the decomposition $\mathcal{H} \oplus \mathcal{V}$ is $\bar{\nabla}$-parallel the terms on the left hand-side vanish due to the symmetries (1.10) of the curvature tensor $\bar{R}$. The right hand-side is determined with the help of Lemma 5.1 and Corollary 5.2. If we apply $\bar{\nabla}$ to the formula (5.2) we obtain by $\bar{\nabla}(\nabla J)=0$ the identity $g\left(\bar{\nabla}_{U}(\bar{R})\left(V_{1}, V_{2}, V_{3}\right),\left(\nabla_{X} J\right) Z\right)=0$ with $Z=J Y$. This yields the proposition using Lemma 5.3 (ii) part d).

\footnotetext{
${ }^{1}$ Here we use $`$ for the inverse construction of $\uparrow$.
} 
5.3. Six-dimensional nearly pseudo-Kähler manifolds. Before analyzing the general case we first focus on dimension six.

Lemma 5.7. On a six-dimensional nearly pseudo-Kähler manifold $\left(M^{6}, J, g\right)$ the integral manifolds of the foliation $\mathcal{V}$ have Gaussian curvature $4 \alpha$ and constant curvature $\kappa=4 \alpha$, where $\alpha$ is the type constant.

Recall that the sign of $\alpha$ is completely determined by the signature of the metric $g$, cf. section 2.1.

Proof. Let $X$ and $V$ be (local) vector fields of constant length in $\mathcal{H}$ and $\mathcal{V}$, respectively. Then it follows from equation (1.7) and the skew-symmetry of $\nabla J: g\left(\nabla_{V} V, \nabla_{X} X\right)=g\left(\bar{\nabla}_{V} V, \bar{\nabla}_{X} X\right)=0$. This identity yields

$$
\begin{aligned}
& R(X, V, X, V) \quad=\quad-g\left(\nabla_{V} \nabla_{X}+\nabla_{[X, V]} V, X\right) \\
& \stackrel{N K}{=} \quad g\left(\nabla_{X} V, \nabla_{V} X\right)-\frac{1}{2} g\left(J\left(\nabla_{[X, V]} J\right) V, X\right)
\end{aligned}
$$

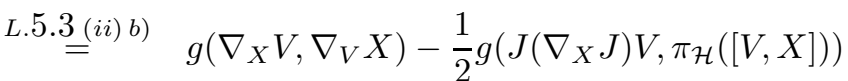

$$
\begin{aligned}
& \stackrel{(*)}{=} \quad g\left(\nabla_{X} V, \nabla_{V} X\right)-\frac{1}{2} g\left(J\left(\nabla_{X} J\right) V, \nabla_{V} X-\frac{1}{2} J\left(\nabla_{X} J\right) V\right) \\
& =\quad g\left(\bar{\nabla}_{X} V, \nabla_{V} X\right)+\frac{1}{4} g\left(\left(\nabla_{X} J\right) V,\left(\nabla_{X} J\right) V\right)
\end{aligned}
$$

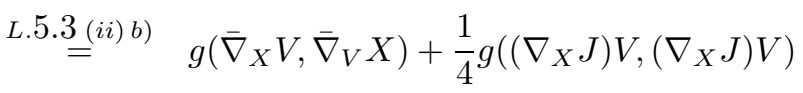

$$
\begin{aligned}
& =\frac{1}{4} g\left(\left(\nabla_{X} J\right) V,\left(\nabla_{X} J\right) V\right) \text {. }
\end{aligned}
$$

At the equality $(*)$ we use Lemma 5.3 (ii) b) which implies, that $\nabla_{V} X=\bar{\nabla}_{V} X+\frac{1}{2} J\left(\nabla_{V} J\right) X \in \mathcal{H}$ to show

$$
\begin{aligned}
\pi_{\mathcal{H}}([V, X]) & =\nabla_{V} X-\pi_{\mathcal{H}}\left(\bar{\nabla}_{X} V\right)-\frac{1}{2} J\left(\nabla_{X} J\right) V \\
& =\nabla_{V} X-\frac{1}{2} J\left(\nabla_{X} J\right) V .
\end{aligned}
$$

Since $M^{6}$ is strict, we obtain $R(X, V, X, V)=\frac{1}{4} \alpha g(X, X) g(V, V)$. In addition it holds $\operatorname{Ric}=5 \alpha g$ which implies

$$
\begin{aligned}
5 \alpha g(V, V) & =g(\operatorname{Ric}(V), V)=g(V, V) R(J V, V, J V, V)+\sum_{i=1}^{4} g\left(e_{i}, e_{i}\right) R\left(e_{i}, V, e_{i}, V\right) \\
& =g(V, V) R(J V, V, J V, V)+\frac{\alpha}{4} \sum_{i=1}^{4} g\left(e_{i}, e_{i}\right)^{2} g(V, V),
\end{aligned}
$$

where $\left\{e_{i}\right\}_{i=1}^{4}$ is an orthogonal frame of $\mathcal{H}$. This equation yields $R(J V, V, J V, V)=4 \alpha$ and it follows that the fibers have Gaussian curvature

$$
K=\frac{R(J V, V, J V, V)}{g(V, V) g(J V, J V)-g(V, J V)^{2}}=4 \alpha
$$

and constant curvature $\kappa=4 \alpha$.

Proposition 5.8. The manifold $(M, J, g)$ is the total space of a pseudo-Riemannian submersion $\pi:(M, g) \rightarrow(N, h)$ where $(N, h)$ is an almost pseudo-hermitian manifold and the fibers are totally geodesic hermitian symmetric spaces. In particular, the fibers are simply connected.

Proof. The foliation which is induced by $\mathcal{V}$ is totally geodesic and each leaf is by Proposition 5.6 a locally hermitian symmetric space of complex dimension one.

It is shown in Lemma 5.7 that each leaf has constant curvature $\kappa$. In the case $\kappa>0$ the leaves are compact and we can apply a result of Kobayashi, cf. [3] 11.26, to obtain that the leaves are simply connected. Since the leaves are also simply connected it follows, that the leaf holonomy is 
trivial and that the foliation comes from a (smooth) submersion (cf. p. 90 of [26]). In the case $\kappa<0$ we observe, that $(M, J,-g)$ is a nearly pseudo-Kähler manifold of constant type $-\alpha$. The same argument shows that the fibers are simply connected.

Lemma 5.9. Let $\left(M^{6}, g, J\right)$ be a strict nearly pseudo-Kähler six-manifold of constant type $\alpha$. For an arbitrary normalized ${ }^{2}$ local vector field $V \in \mathcal{V}$, i.e. $\epsilon_{V}=g(V, V) \in\{ \pm 1\}$, we consider the endomorphisms $\tilde{J}_{1}:=J_{\mid \mathcal{H}}, \tilde{J}_{2}: \mathcal{H} \ni X \mapsto\left(\nabla_{V} J\right) X \in \mathcal{H}$ and $\tilde{J}_{3}=\tilde{J}_{1} \tilde{J}_{2}$. Then the triple $\left(\tilde{J}_{1}, \tilde{J}_{2}, \tilde{J}_{3}\right)$ defines an $\epsilon$-quaternionic triple on $\mathcal{H}$ with $\epsilon_{1}=-1$ and $\epsilon_{2}=\epsilon_{3}=\operatorname{sign}\left(-\alpha \epsilon_{V}\right)$ and it is

$$
\pi^{\mathcal{H}}\left[\left(\nabla_{\chi} \tilde{J}_{i}\right) Y\right]=-\theta_{k}(\chi) \epsilon_{j} \tilde{J}_{j} Y+\theta_{j}(\chi) \epsilon_{k} \tilde{J}_{k} Y
$$

for a cyclic permutation of $i, j, k$ and with $\theta_{1}(\chi)=\operatorname{sign}(\alpha) g\left(J V, \bar{\nabla}_{\chi} V\right), \theta_{2}(\chi)=-\operatorname{sign}(\alpha) \sqrt{|\alpha|} g(V, J \chi)$ and $\theta_{3}(\chi)=\operatorname{sign}(\alpha) \sqrt{|\alpha|} g(V, \chi)$. The sub-bundle of endomorphisms spanned by $\left(\tilde{J}_{1}, \tilde{J}_{2}, \tilde{J}_{3}\right)$ does not depend on the choice of $V$.

Proof. Let $A(X)=\left(\nabla_{V} J\right) X$ for a fixed $V \in \mathcal{V}$ with $\epsilon_{V}=g(V, V) \in\{ \pm 1\}$ and an arbitrary $X \in \mathcal{H}$. Then it is

$$
\begin{aligned}
g\left(A^{2}(X), X\right) & =-g(A(X), A(X))=-g\left(\left(\nabla_{V} J\right) X,\left(\nabla_{V} J\right) X\right) \\
& =-\alpha g(V, V) g(X, X)
\end{aligned}
$$

and we find after polarizing the last expression in $X$ the identity $A^{2}(X)=-\alpha \epsilon_{V} X$.

Furthermore $A$ is a skew-symmetric endomorphism field and in consequence trace-free. Therefore the endomorphism field

$$
\tilde{J}_{2}=\frac{1}{\sqrt{|\alpha|}}\left(\nabla_{V} J\right)
$$

is a hermitian structure if $\alpha \epsilon_{V}>0$ and a para-hermitian structure (since it is trace-free) if $\alpha \epsilon_{V}<0$. Next we set $\tilde{J}_{1}=J_{\mid \mathcal{H}}$ and $\tilde{J}_{3}:=\tilde{J}_{1} \circ \tilde{J}_{2}=-\tilde{J}_{2} \circ \tilde{J}_{1}$, which follows from $(\nabla J) \circ J=-J \circ(\nabla J)$ and observe $\tilde{J}_{3}^{2}=\tilde{J}_{2}^{2}$. Moreover these are (para-)hermitian structures, since $J$ and $\nabla_{V} J$ are skewsymmetric w.r.t. the metric $g$. Hence the triple $\left(\tilde{J}_{1}, \tilde{J}_{2}, \tilde{J}_{3}\right)$ is a (para-)quaternionic triple on $\mathcal{H}$ with $\epsilon_{1}=-1$ and $\epsilon_{2}=\epsilon_{3}=\operatorname{sign}\left(-\alpha \epsilon_{V}\right)$. In the following we suppose, that it holds

$$
\left(\nabla_{X} \tilde{J}_{1}\right) Y \in \mathcal{V}, \text { for } X, Y \in \mathcal{H}
$$

This identity yields $\pi^{\mathcal{H}}\left(\left(\nabla_{X} \tilde{J}_{1}\right) Y\right)=0$ and in consequence it is

$$
\begin{aligned}
\pi^{\mathcal{H}}\left(\left(\nabla_{\chi} \tilde{J}_{1}\right) Y\right) & =\epsilon_{V} g(V, \chi)\left(\nabla_{V} \tilde{J}_{1}\right) Y+\epsilon_{V} g(J V, \chi)\left(\nabla_{J V} \tilde{J}_{1}\right) Y \\
& =\epsilon_{V} g(V, \chi)\left(\nabla_{V} \tilde{J}_{1}\right) Y+\epsilon_{V} g(V, J \chi) J\left(\nabla_{V} \tilde{J}_{1}\right) Y \\
& =\epsilon_{V} \sqrt{|\alpha|} g(V, \chi) \tilde{J}_{2} Y+\epsilon_{V} \sqrt{|\alpha|} g(V, J \chi) \tilde{J}_{3} Y \stackrel{! !}{=}-\theta_{3}(\chi) \epsilon_{2} \tilde{J}_{2} Y+\theta_{2}(\chi) \epsilon_{3} \tilde{J}_{3} Y
\end{aligned}
$$

where we have to define

$$
\begin{aligned}
& \theta_{2}(\chi)=\epsilon_{3} \epsilon_{V} \sqrt{|\alpha|} g(V, J \chi)=-\operatorname{sign}(\alpha) \sqrt{|\alpha|} g(V, J \chi), \\
& \theta_{3}(\chi)=-\epsilon_{2} \epsilon_{V} \sqrt{|\alpha|} g(V, \chi)=\operatorname{sign}(\alpha) \sqrt{|\alpha|} g(V, \chi) .
\end{aligned}
$$

Further we compute using the relation (1.7) for $\nabla$ and $\bar{\nabla}$

$$
\left(\nabla_{\chi} \tilde{J}_{2}\right) Y=\bar{\nabla}_{\chi}\left(\tilde{J}_{2} Y\right)-\tilde{J}_{2} \bar{\nabla}_{\chi} Y+\frac{1}{2 \sqrt{|\alpha|}}\left[J\left(\nabla_{\chi} J\right),\left(\nabla_{V} J\right)\right] Y, \text { for } \chi \in T M, Y \in \mathcal{H}
$$

and get using $\bar{\nabla}(\nabla J)=0$

$$
\begin{aligned}
\pi^{\mathcal{H}}\left[\left(\bar{\nabla}_{\chi} \tilde{J}_{2}\right) Y\right] & =\frac{1}{\sqrt{|\alpha|}} \pi^{\mathcal{H}}\left[\left(\bar{\nabla}_{\chi}\left(\nabla_{V} J\right)\right) Y\right]=-\frac{1}{\sqrt{|\alpha|}} \pi^{\mathcal{H}}\left[\left(\nabla_{\bar{\nabla}_{\chi} V} J\right) Y\right] \\
& =-\frac{1}{\sqrt{|\alpha|}} \epsilon_{V} g\left(J V, \bar{\nabla}_{\chi} V\right)\left(\nabla_{J V} J\right) Y=\frac{1}{\sqrt{|\alpha|}} \epsilon_{V} g\left(J V, \bar{\nabla}_{\chi} V\right) J\left(\nabla_{V} J\right) Y,
\end{aligned}
$$

\footnotetext{
${ }^{2}$ Constant non-zero length suffices.
} 
where we recall that $\bar{\nabla}_{\chi} V \in \mathcal{V}$ has no part parallel to $V$. Due to equation (5.3) and Lemma 5.3 (ii) the last term of (5.4) lies in $\mathcal{V}$ if $\chi$ is in $\mathcal{H}$ and vanishes if $\chi$ is a multiple of $J V$. For $\chi=V$ we get $\left[J\left(\nabla_{V} J\right),\left(\nabla_{V} J\right)\right] Y=2|\alpha| \tilde{J}_{3} \tilde{J}_{2} Y=2 \epsilon_{2}|\alpha| \tilde{J}_{1} Y$. This shows

$$
\begin{aligned}
\pi^{\mathcal{H}}\left[\left(\nabla_{\chi} \tilde{J}_{2}\right) Y\right] & =\epsilon_{2} \sqrt{|\alpha|} \epsilon_{V} g(V, \chi) \tilde{J}_{1} Y+\epsilon_{V} g\left(J V, \bar{\nabla}_{\chi} V\right) \tilde{J}_{3} Y \\
& =-\theta_{1}(\chi) \epsilon_{3} \tilde{J}_{3} Y+\theta_{3}(\chi) \epsilon_{1} \tilde{J}_{1} Y
\end{aligned}
$$

if we set $\theta_{1}(\chi)=-\epsilon_{3} \epsilon_{V} g\left(J V, \bar{\nabla}_{\chi} V\right)=\operatorname{sign}(\alpha) g\left(J V, \bar{\nabla}_{\chi} V\right)$. It remains to differentiate the third (para-)complex structure:

$$
\left(\nabla_{\chi} \tilde{J}_{3}\right) Y=\bar{\nabla}_{\chi}\left(\tilde{J}_{3} Y\right)-\tilde{J}_{3} \bar{\nabla}_{\chi} Y+\frac{1}{2 \sqrt{|\alpha|}}\left[J\left(\nabla_{\chi} J\right), J\left(\nabla_{V} J\right)\right] Y .
$$

Again one obtains using $\bar{\nabla} J=0$ and $\bar{\nabla}(\nabla J)=0$ :

$$
\begin{aligned}
\pi^{\mathcal{H}}\left[\left(\bar{\nabla}_{\chi} \tilde{J}_{3}\right) Y\right] & =\frac{1}{\sqrt{|\alpha|}} \pi^{\mathcal{H}}\left[\left(\bar{\nabla}_{\chi}\left(J \nabla_{V} J\right)\right) Y\right]=-\frac{1}{\sqrt{|\alpha|}} \pi^{\mathcal{H}}\left[J\left(\nabla_{\bar{\nabla}_{\chi} V} J\right) Y\right] \\
& =-\frac{1}{\sqrt{|\alpha|}} \epsilon_{V} g\left(J V, \bar{\nabla}_{\chi} V\right) J\left(\nabla_{J V} J\right) Y=\theta_{1}(\chi) \epsilon_{2} \tilde{J}_{2} Y .
\end{aligned}
$$

The last term of equation (5.5) lies (with the help of equation (5.3) and Lemma 5.3 (ii)) in $\mathcal{V}$ for $\chi \in \mathcal{H}$ and vanishes if $\chi$ is a multiple of $V$. For $\chi=J V$ we compute $\left[J\left(\nabla_{J V} J\right), J\left(\nabla_{V} J\right)\right] Y=$ $\left[\left(\nabla_{V} J\right), J\left(\nabla_{V} J\right)\right] Y=2|\alpha| \tilde{J}_{2} \tilde{J}_{3} Y=-2 \epsilon_{3}|\alpha| \tilde{J}_{1} Y$. This yields

$$
\pi^{\mathcal{H}}\left[\left(\nabla_{\chi} \tilde{J}_{3}\right) Y\right]=-\theta_{2}(\chi) \epsilon_{1} \tilde{J}_{1} Y+\theta_{1}(\chi) \epsilon_{2} \tilde{J}_{2} Y
$$

Given a second section $U$ in $\mathcal{V}$ with $g(U, U)=g(V, V)$ one has $U=a V+b J V$ for real functions $a, b$ with $a^{2}+b^{2}=1$. Using this one easily sees that the triple induced by $V$ and the one by $U$ (locally) spans the same sub-bundle $Q$ of endomorphisms of $\mathcal{H}$.

Lemma 5.10. Let $\left(M^{6}, g, J\right)$ be a strict nearly pseudo-Kähler six-manifold of constant type $\alpha$. Let $s: U \subset N \rightarrow M$ be a (local) section ${ }^{3}$ of $\pi$ on some open set $U$. Define $\phi$ by

$$
\phi=s_{*} \circ \pi_{*}: \mathcal{H}_{s(n)} \stackrel{\pi_{*}}{\rightarrow} T_{n} N \stackrel{s_{*}}{\rightarrow} s_{*}\left(T_{n} N\right) \subset T_{s(n)} M, \text { for } n \in N
$$

and set $J_{i \mid n}:=\pi_{*} \circ \tilde{J}_{i \mid s(n)} \circ\left(\pi_{* \mid \mathcal{H}}\right)^{-1}$ for $i=1, \ldots, 3$, where $\tilde{J}_{i}$ are defined in Lemma 5.9. Then $\left(J_{1}, J_{2}, J_{3}\right)$ defines a local $\epsilon$-quaternionic basis preserved by the Levi-Civita connection $\nabla^{N}$ of $N$.

Proof. We choose $U$ such that the section $s$ is a diffeomorphism onto $W=s(U)$ and a vector field $V$ in $\mathcal{V}$ defined on a subset containing $W$. As $\pi$ is a pseudo-Riemannian submersion we obtain from $\pi_{*} \circ s_{*}=\mathbb{1}$ that $s$ is an isometry from $U$ onto $W$. Therefore it holds $s_{*}\left(\nabla_{X}^{N} Y\right)=\pi^{s_{*} T N}\left[\nabla_{s_{*} X} s_{*} Y\right]$ which yields $\nabla_{X}^{N} Y=\pi_{*}\left(\nabla_{s_{*} X} s_{*} Y\right)$ and

$$
\left(\pi_{* \mid \mathcal{H}}\right)^{-1}\left(\nabla_{X}^{N} Y\right)=\pi^{\mathcal{H}}\left(\nabla_{s_{*} X} s_{*} Y\right) .
$$

For convenience let us identify $U$ and $W$ or in other words consider $s$ as the inclusion $W \subset M$. Then the projection on $s_{*} T N$ is $\phi=s_{*} \pi_{*}=\pi_{*} \mid \mathcal{H}$. Moreover we need the (tensorial) relation

$$
\nabla_{X}^{N}\left(\pi_{*} Z\right)-\pi_{*} \pi^{\mathcal{H}}\left(\nabla_{X}^{M} Z\right)=0 \text { or equivalently } \nabla_{X}^{N} \tilde{Z}-\pi_{*} \pi^{\mathcal{H}}\left(\nabla_{X}^{M} \phi^{-1} \tilde{Z}\right)=0,
$$

which can be directly checked for basic vector fields. Using this identity we get for $i=1, \ldots, 3$

$$
\begin{aligned}
\nabla_{X}^{N}\left(J_{i} Y\right) & =\nabla_{X}^{N}\left(\phi \tilde{J}_{i} \phi^{-1} Y\right)=\phi \nabla_{X}^{M}\left(\tilde{J}_{i} \phi^{-1} Y\right)=\phi\left(\nabla_{X}^{M} \tilde{J}_{i}\right) \phi^{-1} Y+\phi \tilde{J}_{i} \nabla_{X}^{M}\left(\phi^{-1} Y\right) \\
& =\phi\left(\nabla_{X}^{M} \tilde{J}_{i}\right) \phi^{-1} Y+\phi \tilde{J}_{i} \phi^{-1} \nabla_{X}^{N} Y=\phi\left(\nabla_{X}^{M} \tilde{J}_{i}\right) \phi^{-1} Y+J_{i} \nabla_{X}^{N} Y,
\end{aligned}
$$

which reads $\left(\nabla_{X}^{N} J_{i}\right) Y=\phi\left(\nabla_{X}^{M} \tilde{J}_{i}\right) \phi^{-1} Y$. This finishes the proof, since the right hand-side is completely determined by Lemma 5.9, Therefore we have checked the condition (4.10), i.e. the manifold $N$ is endowed with a parallel skew-symmetric (para-)quaternionic structure, see also [3] 10.32 and 14.36 .

\footnotetext{
${ }^{3}$ Local sections exist, since $\pi$ is locally trivial [3] 9.3 .
} 
5.4. General dimension. In the last section we have seen that in dimension six the tensor $\nabla_{V} J$ induces a (para-)complex structure on $\mathcal{H}$. This motivates the following definition.

Definition 5.11. The foliation induced by $T M=\mathcal{H} \oplus \mathcal{V}$ is called of twistorial type if for all $p \in M$ there exists a $V \in \mathcal{V}_{p}$ such that the endomorphism

$$
\nabla_{V} J: \mathcal{H}_{p} \rightarrow \mathcal{H}_{p}
$$

is injective.

Obviously, if $\nabla_{V} J$ defines a (para-)complex structure, then the foliation is of twistorial type.

Proposition 5.12.

(a) If the metric induced on $\mathcal{H}$ is definite, then the foliation is of twistorial type.

(b) If the foliation is of twistorial type, then for all $p \in M$ and all $0 \neq U \in \mathcal{V}_{p}$ the endomorphism is injective.

$$
\nabla_{U} J: \mathcal{H}_{p} \rightarrow \mathcal{H}_{p}
$$

(c) It holds with $A:=\nabla_{V} J$ for some vector field $V$ in $\mathcal{V}$ of constant length and for vector fields $X \in \mathcal{H}$ and $\chi \in T M$

$$
\bar{\nabla}_{\chi}\left(A^{2}\right) X=0
$$

Further it holds $\left[A^{2},\left(\nabla_{U} J\right)\right]=0$ for all $U \in \mathcal{V}$ and

$$
\nabla_{U}\left(A^{2}\right) X=0
$$

for vector fields $U$ in $\mathcal{V}$.

Proof. Part (a) follows from $\left(\nabla_{V} J\right) X \in \mathcal{H}$ for $X \in \mathcal{H}$ and $V \in \mathcal{V}$, cf. Lemma 5.3 (i). For (b) we observe, that if $\nabla_{V} J$ is injective so is $\nabla_{J V} J=-J \nabla_{V} J$. As $\mathcal{V}$ is of dimension two $\{V, J V\}$ with $V \neq 0$ is an orthogonal basis. With $a, b \in \mathbb{R}$ it follows $g\left(\left(a \nabla_{V} J+b \nabla_{J V} J\right) X,\left(a \nabla_{V} J+b \nabla_{J V} J\right) X\right)=$ $\left(a^{2}+b^{2}\right) g\left(\left(\nabla_{V} J\right) X,\left(\nabla_{V} J\right) X\right)$, which yields, that $\nabla_{a V+b J V} J: \mathcal{H}_{p} \rightarrow \mathcal{H}_{p}$ is injective since $a \neq 0$ or $b \neq 0$. It remains to prove part (c). We first observe, that, since $V$ has constant length and since $\bar{\nabla}$ is a metric connection and preserves $\mathcal{V}$, it follows $\bar{\nabla}_{\chi} V=\alpha(\chi) J V$ for some one-form $\alpha$. From $\bar{\nabla}(\nabla J)=0$ we obtain

$$
\left(\bar{\nabla}_{\chi} A\right) X=\left(\bar{\nabla}_{\chi}\left(\nabla_{V} J\right)\right) X=\left(\nabla_{\bar{\nabla}_{\chi} V} J\right) X=\alpha(\chi)\left(\nabla_{J V} J\right) X=-\alpha(\chi) J A X
$$

and we compute using $\{A, J\}=0$

$$
\bar{\nabla}_{\chi}\left(A^{2}\right) X=A\left(\bar{\nabla}_{\chi} A\right) X+\left(\bar{\nabla}_{\chi} A\right) A X=-\alpha(\chi)\left[A(J(A X))+J A^{2} X\right]=0 .
$$

The expression $\left[A^{2},\left(\nabla_{U} J\right)\right]=0$ is tensorial in $U$ and vanishes for $U=V$. Therefore we only need to compute $\left[A^{2},\left(\nabla_{J V} J\right)\right]=-\left[A^{2}, J\left(\nabla_{V} J\right)\right]=-J\left[A^{2},\left(\nabla_{V} J\right)\right]=0$, where we used that $A^{2}$ commutes with $J$. This implies

$$
\nabla_{U}\left(A^{2}\right) X=\bar{\nabla}_{U}\left(A^{2}\right) X+\frac{1}{2}\left[J\left(\nabla_{U} J\right), A^{2}\right] X=-\frac{1}{2}\left[\left(\nabla_{J U} J\right), A^{2}\right] X=0
$$

and proves part (c).

In the following $V$ is a local vector field of constant length $\epsilon_{V}=g(V, V) \in\{ \pm 1\}$.

We denote by $\Omega$ the curvature form of the connection induced by $\bar{\nabla}$ on the (complex) line bundle $\mathcal{V}$, which is given by

$$
\bar{R}(X, Y) V=\Omega(X, Y) J V, \text { for } X, Y \in T M, V \in \mathcal{V} .
$$

Proposition 5.13. If the foliation is of twistorial type,

(i) then the endomorphism $A:=\nabla_{V} J_{\mid \mathcal{H}}$ satisfies $A^{2}=\kappa \epsilon_{V} \mathbb{1}_{\mathcal{H}}$ for some real constant $\kappa \neq 0$ and

$$
\Omega=-2 \kappa\left(2 \omega^{\mathcal{V}}-\omega^{\mathcal{H}}\right),
$$

where $\omega^{\mathcal{H}}(X, Y)=g(X, J Y)$ is the restriction of the fundamental two-form $\omega$ to $\mathcal{H}$;

(ii) for $X, Y$ in $\mathcal{H}$ it is $\left(\nabla_{X} J\right) Y \in \mathcal{V}$.

The proof of this proposition is divided in several steps. 


\section{Lemma 5.14.}

(i) For $X, Y$ in $\mathcal{H}$ and $V$ in $\mathcal{V}$ it is $\bar{R}(X, Y, V, J V)=-2 g\left(\left(\nabla_{V} J\right)^{2} X, J Y\right)$.

(ii) For a given $X$ in $\mathcal{H}$ and $V$ in $\mathcal{V}$ it follows $\bar{R}(X, V, V, J V)=0$.

Proof. (i) Since $\mathcal{H}$ is $\bar{\nabla}$-parallel we obtain, that $\underset{X Y V}{\sigma} \bar{R}(X, Y, V, J V)=\bar{R}(X, Y, V, J V)$. This is the left hand-side of the first Bianchi identity (1.12) . The right hand-side reads

$$
\begin{aligned}
{ }_{X Y V}^{\sigma} g\left(\left(\nabla_{X} J\right) Y,\left(\nabla_{V} J\right) J V\right) & =-g\left(\left(\nabla_{V} J\right) X,\left(\nabla_{Y} J\right) J V\right)-g\left(\left(\nabla_{Y} J\right) V,\left(\nabla_{X} J\right) J V\right) \\
& =-2 g\left(\left(\nabla_{V} J\right)^{2} X, J Y\right) .
\end{aligned}
$$

(ii) From the symmetries (1.10) of the curvature tensor $\bar{R}$ it follows $\bar{R}(X, V, V, J V)=\bar{R}(V, J V, X, V)$. This expression vanishes since $\mathcal{H}$ is $\bar{\nabla}$-parallel.

From the last lemma we derive the more explicit expression of the curvature form $\Omega$ :

$$
\Omega=f \omega^{\mathcal{V}}+\epsilon_{V} \alpha,
$$

where $f$ is a smooth function, $\omega^{\mathcal{V}}$ is the restriction of the fundamental two-form $\omega=g(\cdot, J \cdot)$ to $\mathcal{V}$ and $\alpha(X, Y)=-2 g\left(A^{2} X, J Y\right)$.

Lemma 5.15. It holds with $U \in \mathcal{V}$ and $X, Y \in \mathcal{H}$ :

$$
\begin{aligned}
d \omega^{\mathcal{V}}(X, U, J U) & =0, \\
d \alpha(X, U, J U) & =0, \\
d \omega^{\mathcal{V}}(U, X, Y) & \left.=-g\left(\nabla_{U} J\right) X, Y\right), \\
d \alpha(U, X, Y) & =4 g\left(A^{2}\left(\nabla_{U} J\right) X, Y\right) .
\end{aligned}
$$

Proof. For vector fields $A, B, C$ on $M$ it is

$$
\begin{aligned}
\left(\nabla_{A} \omega^{\mathcal{V}}\right)(B, C)= & A \omega^{\mathcal{V}}(B, C)-\omega^{\mathcal{V}}\left(\nabla_{A} B, C\right)-\omega^{\mathcal{V}}\left(B, \nabla_{A} C\right)=A \omega^{\mathcal{V}}(B, C) \\
& -\omega^{\mathcal{V}}\left(\bar{\nabla}_{A} B+\frac{1}{2} J\left(\nabla_{A} J\right) B, C\right)-\omega^{\mathcal{V}}\left(B, \bar{\nabla}_{A} C+\frac{1}{2} J\left(\nabla_{A} J\right) C\right) .
\end{aligned}
$$

If two of them are $X, Y \in \mathcal{H}$ and one is $U \in \mathcal{V}$ we check using the definition of $\omega^{\mathcal{V}}$, the information of Lemma 5.3 and that the decomposition $\mathcal{H} \oplus \mathcal{V}$ is $\bar{\nabla}$-parallel:

$$
\begin{aligned}
& \nabla_{U} \omega^{\mathcal{V}}(X, Y)=0 \\
& \nabla_{X} \omega^{\mathcal{V}}(U, Y)=-\omega^{\mathcal{V}}\left(U, \nabla_{X} Y\right)=-\frac{1}{2} \omega^{\mathcal{V}}\left(U, J\left(\nabla_{X} J\right) Y\right), \\
& \nabla_{Y} \omega^{\mathcal{V}}(X, U)=-\omega^{\mathcal{V}}\left(\nabla_{Y} X, U\right)=-\frac{1}{2} \omega^{\mathcal{V}}\left(J\left(\nabla_{Y} J\right) X, U\right) .
\end{aligned}
$$

By the symmetries of $\omega(J \nabla . J \cdot, \cdot)$ we conclude $d \omega^{\mathcal{V}}(U, X, Y)=-g\left(\left(\nabla_{U} J\right) X, Y\right)$. Next we suppose $X \in \mathcal{H}$ and $U \in \mathcal{V}$ and obtain with Lemma 5.3.

$$
\begin{aligned}
& \nabla_{X} \omega^{\mathcal{V}}(U, J U)=-\omega^{\mathcal{V}}\left(\bar{\nabla}_{X} U, J U\right)-\omega^{\mathcal{V}}\left(U, \bar{\nabla}_{X}(J U)\right)=0, \\
& \nabla_{U} \omega^{\mathcal{V}}(X, J U)=-\frac{1}{2} \omega^{\mathcal{V}}\left(J\left(\nabla_{U} J\right) X, J U\right)-\frac{1}{2} \omega^{\mathcal{V}}\left(X, J\left(\nabla_{U} J\right) J U\right)=0, \\
& \nabla_{J U} \omega^{\mathcal{V}}(U, X)=-\frac{1}{2} \omega^{\mathcal{V}}\left(J\left(\nabla_{J U} J\right) U, X\right)-\frac{1}{2} \omega^{\mathcal{V}}\left(U, J\left(\nabla_{J U} J\right) X\right)=0 .
\end{aligned}
$$

This shows $d \omega^{\mathcal{V}}(X, U, J U)=0$. Let $X, Y \in \mathcal{H}$ and $U \in \mathcal{V}$. From $\alpha(U, \cdot)=0$ we conclude

$$
\begin{aligned}
& \left(\nabla_{U} \alpha\right)(X, Y)=-2\left[g\left(\nabla_{U}\left(A^{2}\right) X, Y\right)+g\left(A^{2} X,\left(\nabla_{U} J\right) Y\right)\right] \stackrel{\sqrt[5.8]{=}}{=}-2 g\left(A^{2} X,\left(\nabla_{U} J\right) Y\right), \\
& \left(\nabla_{X} \alpha\right)(U, Y)=-g\left(A^{2}\left(\nabla_{U} J\right) X, Y\right),\left(\nabla_{Y} \alpha\right)(U, X)=-g\left(A^{2}\left(\nabla_{U} J\right) Y, X\right),
\end{aligned}
$$


which finishes the proof of $d \alpha(U, X, Y)=4 g\left(A^{2}\left(\nabla_{U} J\right) X, Y\right)$, since $\left[A^{2}, \nabla_{U} J\right]=0$ for all $U \in \mathcal{V}$. We now prove the last identity

$$
\begin{aligned}
& \left(\nabla_{U} \alpha\right)(J U, Y)=-\alpha\left(\bar{\nabla}_{U} J U+\frac{1}{2} J\left(\nabla_{U} J\right) J U, Y\right)-\alpha\left(J U, \bar{\nabla}_{U} Y+\frac{1}{2} J\left(\nabla_{U} J\right) Y\right)=0, \\
& \left(\nabla_{J U} \alpha\right)(Y, U)=-\alpha\left(\bar{\nabla}_{J U} Y+\frac{1}{2} J\left(\nabla_{J U} J\right) Y, U\right)-\alpha\left(Y, \bar{\nabla}_{J U} U+\frac{1}{2} J\left(\nabla_{J U} J\right) U\right)=0, \\
& \left(\nabla_{Y} \alpha\right)(U, J U)=-\alpha\left(\bar{\nabla}_{Y} U+\frac{1}{2} J\left(\nabla_{Y} J\right) U, J U\right)-\alpha\left(U, \bar{\nabla}_{Y} J U+\frac{1}{2} J\left(\nabla_{Y} J\right) J U\right)=0,
\end{aligned}
$$

where we used $\alpha(W, \cdot)=-\alpha(\cdot, W)=0$ for $W \in \mathcal{V}$. This finally shows $d \alpha(X, U, J U)=0$.

Proof. (of the Proposition 5.13) (i) Let $X, Y$ be vector fields in $\mathcal{H}$ and $V$ be a local vector field in $\mathcal{V}$ of constant length. Since $\Omega$ as a curvature form of a (complex) line bundle is closed, we obtain from equation (5.9) $-\epsilon_{V} d \alpha=f d \omega^{\mathcal{V}}+d f \wedge \omega^{\mathcal{V}}$. The equations (5.10) and (5.11) imply $d f_{\mid \mathcal{H}}=0$. This implies $[X, Y] f=0$ and using that $\mathcal{H}$ is $\bar{\nabla}$-parallel we obtain $\left(\bar{\nabla}_{X} Y\right) f=0=\left(\bar{\nabla}_{Y} X\right) f$ which yields finally $0=T^{\bar{\nabla}}(X, Y)(f)=-\left[J\left(\nabla_{X} J\right) Y\right](f)$. By Lemma 5.3 (ii) d) the last equation shows $d f_{\mid \mathcal{V}}=0$. Since $M$ is connected, it follows $f \equiv-\kappa$ for a constant $\kappa$.

Again using $d \Omega(V, X, Y)=0$ equation (5.12) and (5.13) yield for arbitrary $X, Y$

$$
\kappa g\left(\left(\nabla_{V} J\right) X, Y\right)+4 \epsilon_{V} g\left(A^{2}\left(\nabla_{V} J\right) X, Y\right)=0 .
$$

This implies $\left(\nabla_{V} J\right)\left(\kappa \mathbb{1}_{\mathcal{H}}+4 \epsilon_{V} A^{2}\right)=0$. Since the foliation is of twistorial type, it follows

$$
A^{2}=-\epsilon_{V} \frac{\kappa}{4} \mathbb{1}_{\mathcal{H}}=-\epsilon_{V} \alpha \mathbb{1}_{\mathcal{H}}
$$

if we set $4 \alpha=\kappa$ in analgogue to dimension six.

(ii) Since $\Omega$ is closed, it follows from part (i) and $d \omega^{\mathcal{V}}(X, Y, Z)=0$ for $X, Y, Z \in \mathcal{H}$ that it is $d \omega^{\mathcal{H}}(X, Y, Z)=0$. Using $d \omega^{\mathcal{H}}(X, Y, Z)=3 g\left(\left(\nabla_{X} J\right) Y, Z\right)$ yields part (ii).

Proposition 5.16. Let $\left(M^{4 k+2}, g, J\right)$ be a strict nearly pseudo-Kähler manifold of twistorial type. Let $s: U \subset N \rightarrow M$ be a (local) section of $\pi$ on some open set $U$. Define $\phi$ by

$$
\phi=s_{*} \circ \pi_{*}: \mathcal{H}_{s(n)} \stackrel{\pi_{*}}{\rightarrow} T_{n} N \stackrel{s_{*}}{\rightarrow} s_{*}\left(T_{n} N\right) \subset T_{s(n)} M, \text { for } n \in N
$$

and set $J_{i \mid n}:=\pi_{*} \circ \tilde{J}_{i \mid s(n)} \circ\left(\pi_{* \mid \mathcal{H}}\right)^{-1}$ for $i=1, \ldots, 3$, where $\tilde{J}_{i}$ are defined in Lemma 5.9, Then $\left(J_{1}, J_{2}, J_{3}\right)$ defines a local $\epsilon$-quaternionic basis preserved by the Levi-Civita connection $\nabla^{N}$ of $N$.

Proof. The proof of Proposition 5.9 only uses $A^{2}=\kappa \epsilon_{V} \mathbb{1}$ and $\left(\nabla_{X} J\right) Y \in \mathcal{V}$ for $X, Y \in \mathcal{H}$. Therefore we can generalize it by means of Proposition 5.13 to strict nearly pseudo-Kähler manifolds of twistorial type.

5.5. The twistor structure. In this subsection we finally characterize the nearly pseudo-Kähler structures, which are related to the canonical nearly Kähler structure of twistor spaces.

\section{Theorem 5.17.}

(i) The manifold $\left(M, J=\breve{J}, \check{g}=g_{2}\right)$ is a twistor space of a quaternionic pseudo-Kähler manifold, if it is $\epsilon_{V} \alpha>0$.

(ii) The manifold $\left(M, J=\breve{J}, \check{g}=g_{2}\right)$ is a twistor space of a para-quaternionic Kähler manifold, if it is $\epsilon_{V} \alpha<0$.

Proof. Denote by $\pi^{\mathcal{Z}}: \mathcal{Z} \rightarrow N$ the twistor space of the manifold $N$ endowed with the parallel skewsymmetric (para-)quaternionic structure constructed from the foliation $\pi: M \rightarrow N$ of twistorial type, cf. Proposition 5.9 for dimension six and Proposition 5.16 for general dimension. We observe that the restriction of $J$ to $\mathcal{H}$ yields a (smooth) map

$$
\varphi: M \rightarrow \mathcal{Z}, \quad m \mapsto d \pi_{m} \circ J_{m \mid \mathcal{H}} \circ\left(d \pi_{m \mid \mathcal{H}}\right)^{-1}=: j_{\pi(m)},
$$

which by construction satisfies $\pi^{\mathcal{Z}} \circ \varphi=\pi$ and as a consequence $d \pi^{\mathcal{Z}} \circ d \varphi=d \pi$. Since $\pi$ and $\pi^{\mathcal{Z}}$ are pseudo-Riemannian submersions, the last equation implies that $d \varphi$ induces an isometry of the according horizontal distributions and maps the vertical spaces into each other. Let us determine 
the differential of $\varphi$ on $\mathcal{V}$.

Claim: For $V \in \mathcal{V}$ one has

$$
\begin{aligned}
d \varphi(V) & =2 d \pi \circ\left(\nabla_{V} J\right) \circ\left(d \pi_{\mid \mathcal{H}}\right)^{-1}, \\
d \varphi(J V) & =2 d \pi \circ\left(\nabla_{J V} J\right) \circ\left(d \pi_{\mid \mathcal{H}}\right)^{-1}=-2 d \pi \circ J\left(\nabla_{V} J\right) \circ\left(d \pi_{\mid \mathcal{H}}\right)^{-1} .
\end{aligned}
$$

To prove the claim we consider a (local) vector field $V \in \mathcal{V}$ and a (local) integral curve $\gamma$ of $V$ on some interval $I \ni 0$ with $\gamma(0)=m$. Let $X$ be a vector field in $N$. Denote by $\tilde{X}$ the horizontal lift of $X$. The Lie transport of $\tilde{X}$ along the vertical curve $\gamma$ projects to $X$, i.e. it holds $d \pi_{\gamma(t)}(\tilde{X})=X$ for all $t \in I$ and in consequence $\left(d \pi_{\gamma(t) \mid \mathcal{H}}\right)^{-1} X=\tilde{X}$. In other words $d \pi$ commutes with this Lie transport, which implies

$$
d \varphi(V) X=d \pi\left(\left(\mathcal{L}_{V} J\right) \tilde{X}\right),
$$

as one directly checks using basic vector fields. Therefore we need to determine the Lie-derivative $\mathcal{L}$ of $J$ :

$$
\begin{aligned}
\pi^{\mathcal{H}}\left(\left(\mathcal{L}_{V} J\right) \tilde{X}\right) & =\pi^{\mathcal{H}}([V, J \tilde{X}]-J[V, \tilde{X}]) \\
& =\pi^{\mathcal{H}}\left(\nabla_{V}(J \tilde{X})-\nabla_{J \tilde{X}} V-J \nabla_{V} \tilde{X}+J \nabla_{\tilde{X}} V\right) \\
& =\pi^{\mathcal{H}}\left(\left(\nabla_{V} J\right) \tilde{X}-\frac{1}{2} J\left(\nabla_{J \tilde{X}} J\right) V+\frac{1}{2} J\left(J\left(\nabla_{\tilde{X}} J\right)\right) V\right) \\
& =2\left(\nabla_{V} J\right) \tilde{X} .
\end{aligned}
$$

This shows $d \varphi(V)=2 d \pi \circ\left(\nabla_{V} J\right) \circ\left(d \pi_{\mid \mathcal{H}}\right)^{-1}$, which implies $d \varphi(J V)=2 d \pi \circ\left(\nabla_{J V} J\right) \circ\left(d \pi_{\mid \mathcal{H}}\right)^{-1}=$ $-2 d \pi \circ J\left(\nabla_{V} J\right) \circ\left(d \pi_{\mid \mathcal{H}}\right)^{-1}$. Given a local section $s: N \rightarrow M$ and the associated adapted frame of the (para-)quaternionic structure it follows that $\varphi \circ s$ is $J_{1}, d \varphi(V)$ is related to $J_{2}$ and $d \varphi(J V)$ to $-J_{3}$ which span the tangent space of the fiber $F_{\pi(m)}=S^{2}$ in $\varphi(m)$. The complex structure of $\mathcal{Z}$ maps $J_{2}$ to $J_{3}$. Hence $d \varphi$ is complex linear for the opposite complex structure $\check{J}$ on $M$. Further one sees in this local frame that $\varphi$ maps horizontal part into horizontal part. Therefore $\varphi$ is an isometry for the metric $\check{g}=g_{2}$, i.e. the parameter $t=2$ in the canonical variation of the metric $g$. This means that $\left(M, \breve{J}, \check{g}=g_{2}\right)$ is isometrically biholomorph to $\mathcal{Z}$.

Combining Theorem 2.13 and Theorem 5.17 we obtain Theorem A.

\section{REFERENCES}

[1] D. Alekseevsky, Classification of quaternionic spaces with transitive solvable group of motions, Izv. Akad. Nauk SSSR Ser. Mat. 39 (1975), no. 2, 315-362.

[2] D. Alekseevsky, V. Cortés, The twistor spaces of a para-quaternionic Kähler manifold, Osaka J. Math. 45 (2008), no. 1, 215-251.

[3] A. Besse, Einstein manifolds, Springer (1987).

[4] N. Blažić, Paraquaternionic projective space and pseudo-Riemannian geometry, Publications de l'Institut Mathématique Nouvelle série, tome 60 (74) (1996), 101-107.

[5] F. Belgun, A. Moroianu, Nearly Kähler 6-manifolds with reduced holonomy, Ann. Global Anal. Geom. 19 (2001), no. 4, 307-319.

[6] V. Cortés, Alekseevskian spaces, Diff. Geom. Appl. 6 (1996), no. 2, 129-168.

[7] V. Cortés, Th. Leistner, L. Schäfer, F. Schulte-Hengesbach, Half-flat Structures and Special Holonomy, arXiv:0907.1222

[8] V. Cortés, L. Schäfer, Flat nearly Kähler manifolds, Ann. Global Anal. Geom. 32 (2007), no. 4, 379-389.

[9] V. Cortés, L. Schäfer, Geometric Structures on Lie groups with flat bi-invariant metric, J. Lie Theory 19 (2009), 423-437.

[10] Th. Friedrich, S. Ivanov, Parallel spinors and connections with skew-symmetric torsion in string theory, Asian J. Math. 6 (2002), no. 2, 303-335.

[11] S. Ferrara, S. Sabharwal, Quaternionic manifolds for type II superstring vacua of Calabi-Yau spaces, Nucl. Phys. B 332 (1990), 317-332.

[12] A. Gray, The structure of nearly Kähler manifolds, Math. Ann. 223 (1976), no. 3, 233-248.

[13] A. Gray, Riemannian manifolds with geodesic symmetries of order 3, J. Diff. Geom. 7 (1972), 343-369.

[14] I. Kath, Killing Spinors on Pseudo-Riemannian Manifolds, Habilitationsschrift an der HumboldtUniversität zu Berlin (1999). 
[15] S. Kobayashi, On compact Kähler manifolds with positive definite Ricci tensor, Ann. of Math. 74 (1961), $570-574$.

[16] S. Kobayashi, K. Nomizu, Foundations of differential geometry, Vol. I/II Interscience Publishers John Wiley \& Sons (1969).

[17] C. LeBrun, On complete quaternionic-Kähler manifolds, Duke Math. J. 63 (1991), no.3, 723-743.

[18] H. B. Lawson, M.-L.Michelson, Spin geometry, Princeton University Press, (1989).

[19] P.-A. Nagy, On nearly-Kähler geometry, Ann. Global Anal. Geom. 22 (2002), no. 2, 167-178.

[20] P.-A. Nagy, Nearly Kähler geometry and Riemannian foliations, Asian J. Math. 6 (2002), no. 3, $481-504$.

[21] B. O'Neill, The fundamental equations of a submersion, Mich. Math. J. 13 (1966), 459-469.

[22] J. A. Wolf, Complex homogeneous contact manifolds and quaternionic symmetric spaces, J. Math. Mech. 14 (1965) 1033-1047.

[23] L. Schäfer, F. Schulte-Hengesbach, Nearly pseudo-Kähler and nearly para-Kähler six-manifolds, arXiv:0912.3271 to appear in Handbook of pseudo-Riemannian geometry and supersymmetry, ed. by V. Cortés, IRMA Lect. Math. Theor. Phys. 16 (2010).

[24] L. Schäfer, $t t^{*}$-geometry on the tangent bundle of an almost complex manifold, J. Geom. Phys. 57 (2007), no. 3, 999-1014.

[25] L. Schäfer, Para-tt*-bundles on the tangent bundle of an almost para-complex manifold, Ann. Global Anal. Geom.32 (2007), no. 2, 125-145.

[26] R. W. Sharpe, Differential geometry, Cartan's generalization of Klein's Erlangen program, Springer (1997).

Lars Schäfer, Institut Differentialgeometrie, Leibniz Universität Hannover, Welfengarten 1, D30167 Hannover, Germany

E-mail address: schaefer@math.uni-hannover.de 